TITLE:

\title{
Direct synthesis of highly fused perimidines by copper(I)-catalyzed hydroamination of $2-$ ethynylbenzaldehydes
}

\section{$\operatorname{AUTHOR(S):~}$}

Tokimizu, Yusuke; Ohta, Yusuke; Chiba, Hiroaki; Oishi, Shinya; Fujii, Nobutaka; Ohno, Hiroaki

\section{CITATION:}

Tokimizu, Yusuke ...[et al]. Direct synthesis of highly fused perimidines by copper(l)catalyzed hydroamination of 2-ethynylbenzaldehydes. Tetrahedron 2011, 67(29): 51685175

\section{ISSUE DATE:}

2011-07

URL:

http://hdl.handle.net/2433/143596

\section{RIGHT:}

@ 2011 Elsevier Ltd.; この論文は出版社版でありません。引用の際には 出版社版をご確認ご利用ください。; This is not the published version. Please cite only the published version. 


\section{Graphical Abstract}

Direct synthesis of highly fused perimidines

Leave this area blank for abstract info. by copper(I)-catalyzed hydroamination of 2ethynylbenzaldehydes

Yusuke Tokimizu, Yusuke Ohta, Hiroaki Chiba, Shinya Oishi, Nobutaka Fujii, ${ }^{*}$ Hiroaki Ohno* Graduate School of Pharmaceutical Sciences, Kyoto University, Sakyo-ku, Kyoto 606-8501, Japan

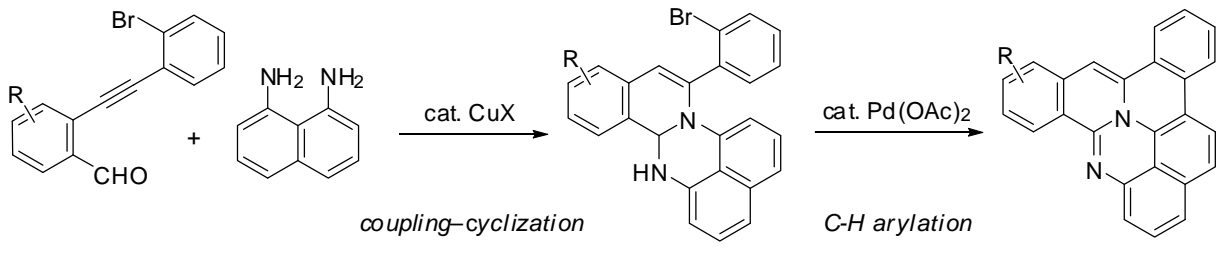




\title{
Direct synthesis of highly fused perimidines by copper(I)-catalyzed hydroamination of 2- ethynylbenzaldehydes
}

\author{
Yusuke Tokimizu, Yusuke Ohta, Hiroaki Chiba, Shinya Oishi, Nobutaka Fujii, ${ }^{*}$ Hiroaki Ohno* \\ Graduate School of Pharmaceutical Sciences, Kyoto University, Sakyo-ku, Kyoto 606-8501, Japan
}

\section{ARTICLE INFO}

\section{ABSTRACT}

\section{Article history:}

\section{Received}

Received in revised form

Accepted

Available online

\section{Keywords:}

C-H arylation

polyarene

isoquinoline

perimidine

multi-component reaction

\section{Introduction}

Because of their charge mobility, highly-conjugated polycyclic compounds are important in practical applications for electronic materials, such as in dye lasers and electroluminescent materials. ${ }^{1-5}$ Compounds with a cyclic amidine moiety, such as fused perimidine derivatives, ${ }^{6}$ are particularly useful because they have high $\pi$-stacking ability, electron affinity, and reduction potential, and can be used as core structures of biologically active compounds. $^{7-9}$

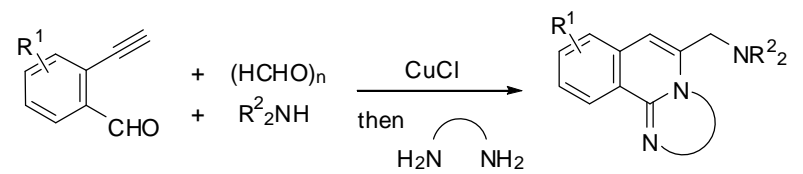<smiles>[X]c1ccccc1C#Cc1ccccc1C=O</smiles>

1<smiles>Nc1cccc2cccc(N)c12</smiles>

cat. CuX<smiles>[R]c1ccccc1C1=Cc2ccccc2[X]C(c2ccccc2[X])N1</smiles>

2<smiles></smiles>

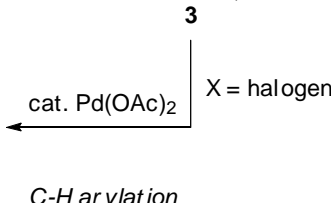

Scheme 1. Strategy for direct synthesis of highly fused perimidines
We have an ongoing program directed toward fused isoquinoline synthesis based on four-component coupling and cyclization cascade (Scheme 1, eq 1). ${ }^{10,11}$ We postulated that a fused perimidine skeleton could be readily constructed by copper-catalyzed annulation of 2-ethynylbenzaldehyde 1 with 1,8-diaminonaphthalene 2 (eq 2). Use of 2-alkynylbenzaldehydes $\mathbf{1}$ bearing an aryl halide moiety ( $\mathrm{X}=$ halogen) with palladiumcatalyzed $\mathrm{C}-\mathrm{H}$ arylation $^{4,5,12-21}$ of the resulting perimidines 3 would provide facile access to a new class of highly fused perimidines 4 .
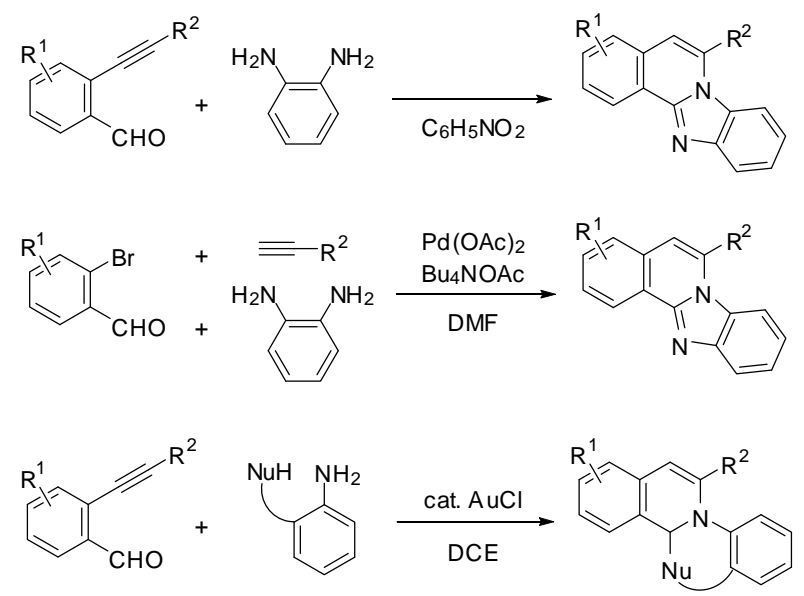

Scheme 2. Related reactions for synthesis of fused isoquinolines

Recently, several syntheses of isoquinoline-fused compounds using a diamine component have been reported (Scheme 2). Dyker et al. used 1,2-phenylenediamine for construction of a benzimidazo[2,1-a]isoquinoline skeleton from 2- 


\section{Tetrahedron}

alkynylbenzaldehydes (eq 3). ${ }^{22,23}$ Yanada et al. reported palladium-catalyzed direct synthesis of benzimidazo[2,1a] isoquinolines through one-pot Sonogashira coupling between 2-bromobenzaldehydes and an alkyne, followed by cyclization with 1,2-phenylenediamine (eq 4). ${ }^{24}$ Sridhar et al. reported a gold-catalyzed reaction of 2-alkynylbenzaldehydes with aniline, which had another nucleophilic functionality such as pyrrole/indole/imidazole rings or amino/sulfonamide/hydroxy groups (eq 5). ${ }^{25}$ However, annulation of 2-alkynylbenzaldehydes with a diamine component in which each of amino groups is located on a different benzene ring is unprecedented. ${ }^{26}$ Here, we report a novel synthesis of highly fused perimidines 4 by coppercatalyzed coupling and cyclization of 2-alkynylbenzaldehydes $\mathbf{1}$ and 1,8-diaminonaphthalene 2 , followed by palladium-catalyzed $\mathrm{C}-\mathrm{H}$ arylation (Scheme 1).

\section{Results and discussion}

The reaction conditions for the copper-catalyzed annulation using 2-[(2-bromophenyl)ethynyl]benzaldehyde $\mathbf{1 a}$ and 1,8diaminonaphthalene 2 were optimized (Table 1). When the aldehyde 1a was treated with 2 in the presence of $\mathrm{CuI}(10$ mol \%) in DMF, the isoquinoline 6 was obtained as the oxidized form in $53 \%$ yield (entry 1 ). The use of dioxane as the solvent at $80{ }^{\circ} \mathrm{C}$ under an $\mathrm{Ar}$ or $\mathrm{O}_{2}$ atmosphere was less effective, and produced the intermediate aminal 5 as the major product (56$67 \%$ yield) along with unoxidized isoquinoline 3a (18-20\% yield, entries 2 and 3). Although the reaction at higher temperature $\left(120^{\circ} \mathrm{C}\right.$ ) increased the yield of 3a to $92 \%$ (entry 4$)$, scaling up the reaction from $0.11 \mathrm{mmol}$ to $1.1 \mathrm{mmol}$ was unsuccessful (entry 5). The use of microwave irradiation solved this problem, and 3a was produced in $91 \%$ yield on the $1.1 \mathrm{mmol}$ scale (entry 6). Because microwave conditions in DMF led to an unsatisfactory result (entry 7), the conditions shown in entry 6 were used for further investigations.

Table 1. Optimization of reaction conditions for the coupling-cyclization ${ }^{\mathrm{a}}$<smiles>O=Cc1ccccc1C#Cc1ccccc1Br</smiles>

$1 \mathrm{a}$<smiles>Brc1ccccc1C#Cc1ccccc1C1Nc2cccc3cccc(c23)N1</smiles>

\begin{tabular}{lllllll}
\hline \multirow{2}{*}{ entry } & \multirow{2}{*}{ solvent } & \multirow{2}{*}{ conditions } & yield $(\%)^{\mathrm{c}}$ & \\
& & & 3a & $\mathbf{5}$ & $\mathbf{6}$ & $\mathbf{7}$ \\
\hline 1 & DMF & $110^{\circ} \mathrm{C}, 20 \mathrm{~h}$ & - & - & 53 & - \\
2 & dioxane & $80^{\circ} \mathrm{C}, 10 \mathrm{~h}$ & 20 & 67 & - & - \\
$3^{\text {d }}$ & dioxane & $80^{\circ} \mathrm{C}, 30 \mathrm{~h}$ & 18 & 56 & 7 & - \\
4 & dioxane & reflux, $12 \mathrm{~h}$ & 92 & - & - & 5 \\
$5^{e}$ & dioxane & reflux, $24 \mathrm{~h}$ & 54 & 34 & - & - \\
$6^{e}$ & dioxane & $\mathrm{MW}^{\mathrm{b}}, 150^{\circ} \mathrm{C}, 60 \mathrm{~min}$ & 91 & - & - & 5 \\
7 & DMF & $\mathrm{MW}^{\mathrm{b}}, 140^{\circ} \mathrm{C}, 60 \mathrm{~min}$ & 56 & - & 7 & 31 \\
\hline
\end{tabular}

${ }^{\text {a } U n l e s s ~ o t h e r w i s e ~ s t a t e d ~ t h e ~ r e a c t i o n s ~ w e r e ~ c o n d u c t e d ~ w i t h ~} \mathbf{1 a}(0.11 \mathrm{mmol})$ and 2 (1.2 equiv) in the presence of $\mathrm{CuI}(10 \mathrm{~mol} \%)$ under $\mathrm{Ar}$.

${ }^{\mathrm{b}} \mathrm{MW}=$ microwave irradiation. ${ }^{\mathrm{c}}$ Isolated yields. ${ }^{\mathrm{d}}$ The reaction was conducted under an $\mathrm{O}_{2}$ atmosphere. ${ }^{\mathrm{e}}$ The reactions were conducted on a $1.1 \mathrm{mmol}$ scale.

The substrate scope of the copper-catalyzed annulation was then examined using substituted 2-alkynylbenzaldehydes $\mathbf{1 b}-\mathbf{h}$ (Table 2). These substrates were readily prepared by Sonogashira coupling of substituted 2-ethynylbenzaldehydes with 2iodobromobenzene (see the experimental section). The substrates 1b-e, bearing an electron-withdrawing (fluoro) or -donating (methyl or methoxy) substituent at the para- or meta-position to the formyl group, underwent the desired annulation under the standard reaction conditions. The corresponding fused perimidines $\mathbf{3 b}-\mathbf{e}$ were produced in good to excellent yields (71$97 \%$, entries 1-4). Substitution with a fluoro group at the ortho position was also tolerated (entry 5). Influence of the benzene substitution at the alkyne terminus with a fluoro or methyl group was less important (entries 6 and 7). The substrates $\mathbf{1 i}$ and $\mathbf{1 j}$ without a bromo substituent also gave the desired products $\mathbf{3 i}$ and 3j in high yields (91\%, entries 8 and 9$)$.

Table 2. Coupling-cyclization of various 2alkynylbenzaldehydes ${ }^{\mathrm{a}}$

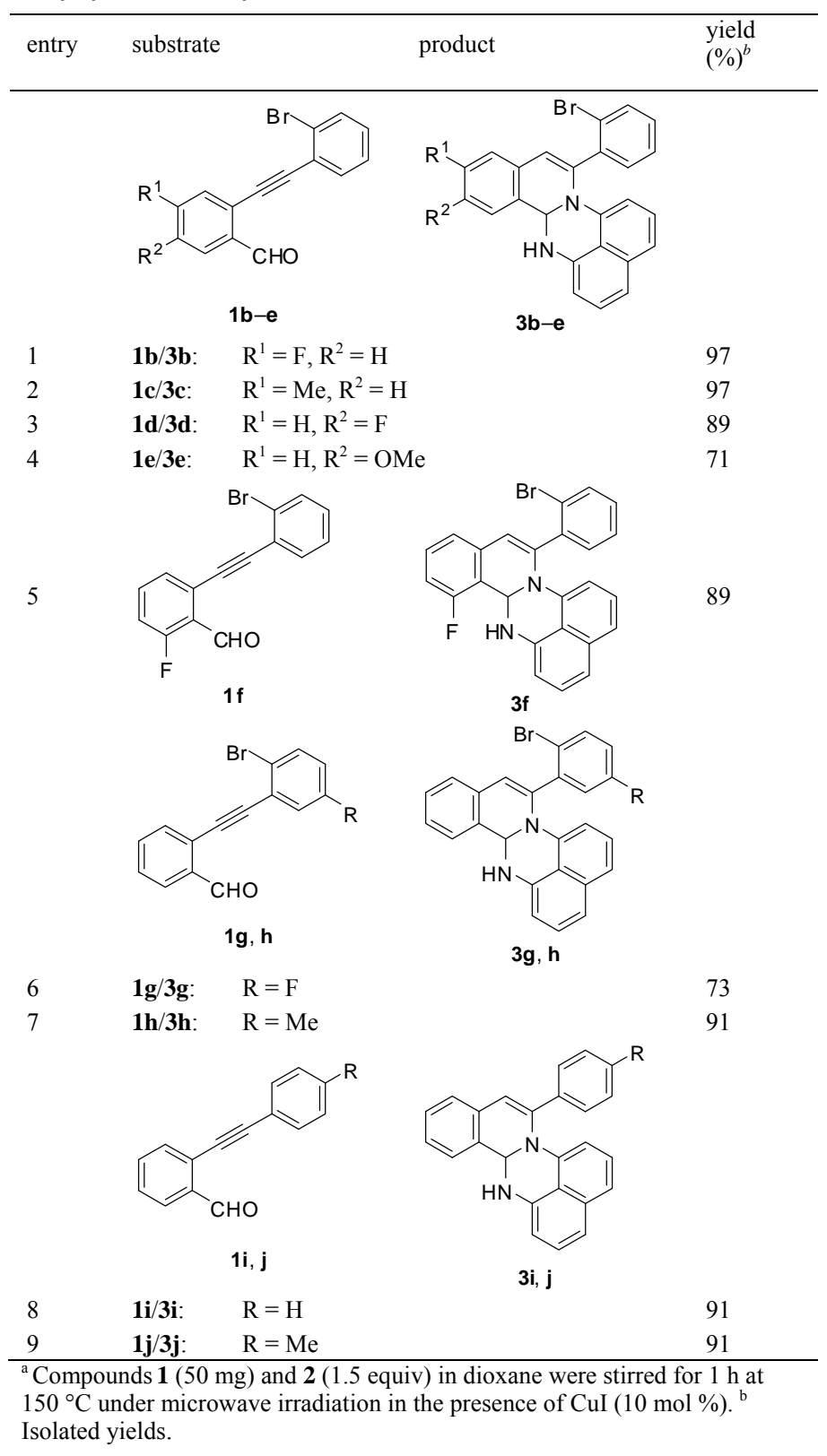


Table 4. Optimization of reaction conditions for palladium-catalyzed C-H arylation ${ }^{\mathrm{a}}$

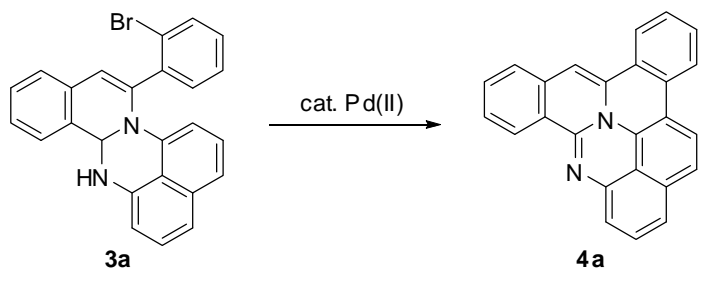

\begin{tabular}{llllllll}
\hline entry & catalyst & ligand & solvent & base & temp. & time & yield (\%) \\
\hline 1 & $\mathrm{Pd}(\mathrm{OAc})_{2}$ & $\mathrm{PPh}_{3}$ & dioxane & $\mathrm{Cs}_{2} \mathrm{CO}_{3}$ & reflux & $5 \mathrm{~h}$ & 40 \\
2 & $\mathrm{Pd}(\mathrm{OAc})_{2}$ & $\mathrm{P}(\mathrm{t}-\mathrm{Bu})_{3} \cdot \mathrm{HBF}_{4}$ & dioxane & $\mathrm{Cs}_{2} \mathrm{CO}_{3}$ & reflux & $5 \mathrm{~h}$ & 43 \\
3 & $\mathrm{Pd}(\mathrm{OAc})_{2}$ & none & dioxane & $\mathrm{Cs}_{2} \mathrm{CO}_{3}$ & reflux & $5 \mathrm{~h}$ & 0 \\
4 & $\mathrm{Pd}(\mathrm{OAc})_{2}$ & $\mathrm{PPh}_{3}$ & dioxane & $\mathrm{Cs}_{2} \mathrm{CO}_{3}$ & $\mathrm{MW}{ }^{\mathrm{c}} 160^{\circ} \mathrm{C}$ & $15 \mathrm{~min}$ & 27 \\
5 & $\mathrm{Pd}(\mathrm{OAc})_{2}$ & $\mathrm{PPh}_{3}$ & $\mathrm{DMF}$ & $\mathrm{Cs}_{2} \mathrm{CO}_{3}$ & $130{ }^{\circ} \mathrm{C}$ & $4 \mathrm{~h}$ & 65 \\
6 & $\mathrm{Pd}(\mathrm{OAc})_{2}$ & $\mathrm{P}_{2}-\mathrm{Bu}_{3} \cdot \mathrm{HBF}_{4}$ & $\mathrm{DMF}$ & $\mathrm{Cs}_{2} \mathrm{CO}_{3}$ & $130^{\circ} \mathrm{C}$ & $6 \mathrm{~h}$ & 56 \\
7 & $\mathrm{Pd}(\mathrm{PPh})_{2} \mathrm{Cl}_{2}$ & $\mathrm{PPh}_{3}$ & $\mathrm{DMF}$ & $\mathrm{Cs}_{2} \mathrm{CO}_{3}$ & $130{ }^{\circ} \mathrm{C}$ & $5 \mathrm{~h}$ & 22 \\
8 & $\mathrm{Pd}(\mathrm{PPh})_{4}$ & $\mathrm{PPh}_{3}$ & $\mathrm{DMF}$ & $\mathrm{Cs}_{2} \mathrm{CO}_{3}$ & $130^{\circ} \mathrm{C}$ & $5 \mathrm{~h}$ & 0 \\
9 & $\mathrm{Pd}(\mathrm{OAc})_{2}$ & $\mathrm{PPh}_{3}$ & toluene & $\mathrm{Cs}_{2} \mathrm{CO}_{3}$ & reflux & $6 \mathrm{~h}$ & $<60^{\mathrm{d}}$ \\
10 & $\mathrm{Pd}(\mathrm{OAc})_{2}$ & $\mathrm{PPh}_{3}$ & $\mathrm{DMSO}$ & $\mathrm{Cs}_{2} \mathrm{CO}_{3}$ & $130^{\circ} \mathrm{C}$ & $6 \mathrm{~h}$ & $<65^{\mathrm{d}}$ \\
11 & $\mathrm{Pd}(\mathrm{OAc})_{2}$ & $\mathrm{PPh}_{3}$ & propan-2-ol & $\mathrm{Cs}_{2} \mathrm{CO}_{3}$ & reflux & $6 \mathrm{~h}$ & 0 \\
12 & $\mathrm{Pd}(\mathrm{OAc})_{2}$ & $\mathrm{PPh}_{3}$ & EtOH & $\mathrm{Cs}_{2} \mathrm{CO}_{3}$ & reflux & $4 \mathrm{~h}$ & 0 \\
13 & $\mathrm{Pd}(\mathrm{OAc})_{2}$ & $\mathrm{PPh}_{3}$ & $\mathrm{DMF}$ & $\mathrm{Na}_{2} \mathrm{CO}_{3}$ & $130{ }^{\circ} \mathrm{C}$ & $4 \mathrm{~h}$ & 39 \\
14 & $\mathrm{Pd}(\mathrm{OAc})_{2}$ & $\mathrm{PPh}_{3}$ & $\mathrm{DMF}$ & $\mathrm{K}_{2} \mathrm{CO}_{3}$ & $130^{\circ} \mathrm{C}$ & $4 \mathrm{~h}$ & $<57^{\mathrm{d}}$
\end{tabular}

${ }^{a}$ All reactions were conducted using 3a $(0.06-0.07 \mathrm{mmol})$ in the presence of a palladium catalyst $(10 \mathrm{~mol} \%)$, ligand ( $\left.25 \mathrm{~mol} \%\right)$, and base (2 equiv).

${ }^{\mathrm{b}}$ Isolated yields. ${ }^{\mathrm{c}} \mathrm{MW}=$ microwave irradiation. ${ }^{\mathrm{d}}$ Contained inseparable impurities.

In order to expand the use of 1,8-diaminonaphthalene 2 as a precursor of other perimidine derivatives, we examined fourcomponent annulation using 2-ethynylbenzaldehyde 8, formaldehyde $\mathbf{9}$, and secondary amine $\mathbf{1 0}$. As shown in Table 3, the reaction with diisopropylamine, piperidine, and morpholine as the secondary amine component produced perimidines 11a-c bearing an aminomethyl group (52-70\% yield).

Table 3. Four-component synthesis of fused perimidines ${ }^{\mathrm{a}}$
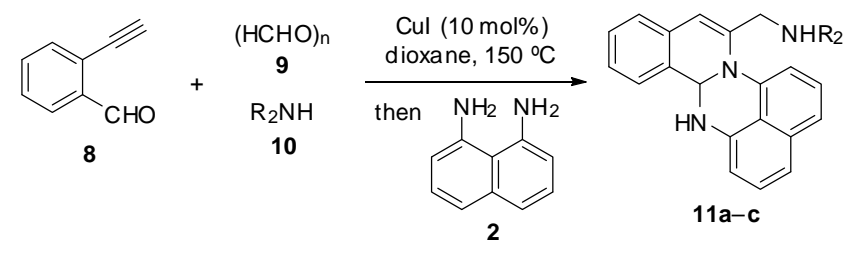

\begin{tabular}{|c|c|c|c|c|}
\hline entry & $\mathrm{R}_{2} \mathrm{NH}$ & conditions $^{b}$ & product & yield $(\%)^{\mathrm{c}}$ \\
\hline 1 & $(i-\mathrm{Pr})_{2} \mathrm{NH}$ & $\mathrm{rt}, 1 \mathrm{~h}$ & 11a & 52 \\
\hline 2 & & $\mathrm{rt}, 6 \mathrm{~h}$ & $11 b$ & 70 \\
\hline 3 & & $\mathrm{rt}, 4 \mathrm{~h}$ & $11 \mathrm{c}$ & 61 \\
\hline
\end{tabular}

${ }^{a}$ After the three-component reaction of $\mathbf{8}(0.23 \mathrm{mmol}), \mathbf{9}$ (2 equiv), and $\mathbf{1 0}(2$ equiv) in the presence of $\mathrm{CuI}(10 \mathrm{~mol} \%)$ in dioxane, 2 (3 equiv) was added and the reaction mixture was stirred for $1 \mathrm{~h}$ at $150{ }^{\circ} \mathrm{C}$ under microwave irradiation. ${ }^{\mathrm{b}}$ Conditions for the reaction of 8 with 9 and 10 . ${ }^{\mathrm{c}}$ Isolated yields.

Next, palladium-catalyzed $\mathrm{C}-\mathrm{H}$ arylation for the synthesis of highly fused perimidines was investigated. Isoquinoline 3a was chosen as the model substrate for optimization of the cyclization conditions (Table 4). When isoquinoline 3a was allowed to react with $\mathrm{Pd}(\mathrm{OAc})_{2}(10 \mathrm{~mol} \%)$ in the presence of $\mathrm{PPh}_{3}(25 \mathrm{~mol} \%)$ and $\mathrm{Cs}_{2} \mathrm{CO}_{3}$ (2 equiv) in dioxane, the desired heptacyclic perimidine $4 \mathbf{a}$ as the oxidized form was obtained in $40 \%$ yield (entry 1 ). The use of $\mathrm{P}(t-\mathrm{Bu})_{3} \cdot \mathrm{HBF}_{4}$ as a ligand slightly improved the yield (43\%, entry 2$)$. Reaction in the absence of phosphine as the ligand (entry 3) or under microwave irradiation at $160{ }^{\circ} \mathrm{C}$ (entry 4) was less effective. DMF was promising as the reaction solvent, and produced $4 \mathbf{a}$ in $65 \%$ yield (entry 5). Among the palladium catalysts and phosphine ligands tested (entries 5-8), $\mathrm{Pd}(\mathrm{OAc})_{2} / \mathrm{PPh}_{3}$ was the most effective in DMF (entry 5). Other solvents (toluene, DMSO, propan-2-ol, and EtOH, entries 9-12) and bases $\left(\mathrm{Na}_{2} \mathrm{CO}_{3}, \mathrm{~K}_{2} \mathrm{CO}_{3}, \mathrm{~K}_{3} \mathrm{PO}_{4}\right.$, and $\mathrm{KOAc}$, entries 13-16) were also examined, and $\mathrm{K}_{3} \mathrm{PO}_{4}$ in DMF was the most effective (78\% yield, entry 15$)$.

Finally, a series of substrates with various substituent patterns were applied to the $\mathrm{C}-\mathrm{H}$ arylation under the optimized conditions for 3a (Table 5). All the substituted substrates $\mathbf{3 b}-\mathbf{h}$ afforded the desired products $\mathbf{4 b}-\mathbf{h}$ as the oxidized form (45-62\% yield, entries 1-7). This result was independent of the substituents on the two benzene rings. The moderate yields were partly because crystallization was required for purification. Poor solubility of $\mathbf{4}$ in various nonpolar or polar solvents, including aromatic solvents, did not allow easy purification by column chromatography. 
These results show that copper-catalyzed annulation of 2alkynylbenzaldenydes $\mathbf{1}$ with 1,8-diaminonaphthalene $\mathbf{2}$, and subsequent palladium-catalyzed arylation provides convenient access to highly fused perimidine derivatives.

Table 5. Synthesis of highly fused perimidines ${ }^{\mathrm{a}}$

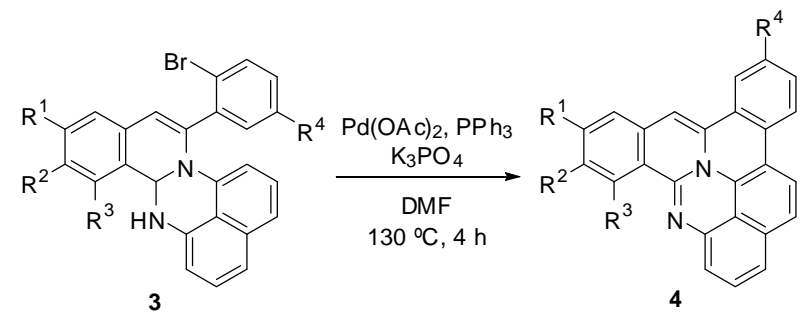

\begin{tabular}{lllllll}
\hline entry & $\begin{array}{l}\text { substrate/ } \\
\text { product }\end{array}$ & $\mathrm{R}^{1}$ & $\mathrm{R}^{2}$ & $\mathrm{R}^{3}$ & $\mathrm{R}^{4}$ & yield (\%) \\
\hline 1 & $\mathbf{3 b} / \mathbf{4 b}$ & $\mathrm{F}$ & $\mathrm{H}$ & $\mathrm{H}$ & $\mathrm{H}$ & 45 \\
2 & $\mathbf{3 c / 4 c}$ & $\mathrm{Me}$ & $\mathrm{H}$ & $\mathrm{H}$ & $\mathrm{H}$ & 53 \\
3 & $\mathbf{3 d} / \mathbf{4 d}$ & $\mathrm{H}$ & $\mathrm{F}$ & $\mathrm{H}$ & $\mathrm{H}$ & 53 \\
4 & $\mathbf{3 e} / \mathbf{4 e}$ & $\mathrm{H}$ & $\mathrm{OMe}$ & $\mathrm{H}$ & $\mathrm{H}$ & 61 \\
5 & $\mathbf{3 f} / \mathbf{4 f}$ & $\mathrm{H}$ & $\mathrm{H}$ & $\mathrm{F}$ & $\mathrm{H}$ & 51 \\
6 & $\mathbf{3 g} / \mathbf{4 g}$ & $\mathrm{H}$ & $\mathrm{H}$ & $\mathrm{H}$ & $\mathrm{F}$ & 49 \\
7 & $\mathbf{3 h} / \mathbf{4 h}$ & $\mathrm{H}$ & $\mathrm{H}$ & $\mathrm{H}$ & $\mathrm{Me}$ & 62
\end{tabular}

${ }^{\mathrm{a}} \mathrm{All}$ reactions were conducted with $3(30 \mathrm{mg})$ in the presence of $\mathrm{K}_{3} \mathrm{PO}_{4}(2$ equiv), $\mathrm{Pd}(\mathrm{OAc})_{2}(10 \mathrm{~mol} \%)$, and $\mathrm{PPh}_{3}(25 \mathrm{~mol} \%)$ in DMF. ${ }^{\mathrm{b}}$ Isolated yields after recrystallization

\section{Conclusions}

Fused perimidine derivatives were synthesized by coppercatalyzed annulation of 2-alkynylbenzaldenydes $\mathbf{1}$ with 1,8diaminonaphthalene 2 . The four-component approach was applied to this reaction to produce perimidine derivatives bearing an aminomethyl group. Palladium-catalyzed C-H arylation of perimidines bearing an aryl bromide moiety produced a new class of highly fused perimidine derivatives in moderate to good yields.

\section{Experimental}

\subsection{General}

${ }^{1} \mathrm{H}$ NMR spectra were recorded using a JEOL AL-500 spectrometer at $500 \mathrm{MHz}$ frequency. Chemical shifts are reported in $\delta(\mathrm{ppm})$ relative to $\mathrm{Me}_{4} \mathrm{Si}$ (in $\mathrm{CDCl}_{3}$ or $\mathrm{CD}_{3} \mathrm{OD}$ ) as internal standard. ${ }^{13} \mathrm{C}$ NMR spectra were recorded using a JEOL AL-500 and referenced to the residual $\mathrm{CHCl}_{3}$ or $\mathrm{MeOH}$ signal. Melting points were measured by a hot stage melting points apparatus (uncorrected). For column chromatography, Wakogel C-300E was employed. Microwave reaction was conducted in a sealed glass vessel (capacity $10 \mathrm{~mL}$ ) using CEM Discover microwave reactor with a run time of no more than $10 \mathrm{~min}$ at below $300 \mathrm{~W}$. The commercially available compounds including $\mathbf{2}, \mathbf{9}, \mathbf{1 0 a}-\mathbf{c}$, 13a-e, and 14 were used without further purification.

The compounds $\mathbf{1 2} \mathbf{a}-\mathbf{e}^{10}$ and $\mathbf{1 2} \mathbf{f}^{27}$ were prepared according to the literature.

\subsection{Preparation of Starting Materials}

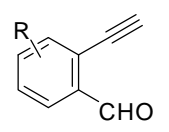

12

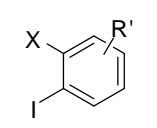

13

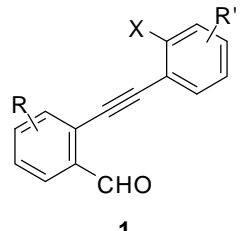

4.2.1. 2-[(2-Bromophenyl)ethynyl]benzaldehyde (1a). A mixture of 2-ethynylbenzaldehyde (12a) (1.00 g, $7.68 \mathrm{mmol})$, 1-bromo-2iodobenzene (13a) (1.18 mL, $9.22 \mathrm{mmol}), \mathrm{CuI}(146 \mathrm{mg}, 0.77$ $\mathrm{mmol}), \mathrm{PdCl}_{2}\left(\mathrm{PPh}_{3}\right)_{2}(107 \mathrm{mg}, 0.15 \mathrm{mmol})$, and $\mathrm{Et}_{3} \mathrm{~N}(15 \mathrm{~mL})$ in THF $(15 \mathrm{~mL})$ was stirred at $80{ }^{\circ} \mathrm{C}$ for $2 \mathrm{~h}$ under argon, and filtrated through a pad of celite. The filtrate was concentrated under reduced pressure and the residue was purified by column chromatography over silica gel with hexane-EtOAc $(15: 1)$ to give 1a $(1.81 \mathrm{~g}, 83 \%)$ as a colorless solid: $\mathrm{mp} 69{ }^{\circ} \mathrm{C} ;{ }^{1} \mathrm{H}$ NMR $\left(500 \mathrm{MHz}, \mathrm{CDCl}_{3}\right) \delta 7.23$ (ddd, $J=8.0,8.0,1.7 \mathrm{~Hz}, 1 \mathrm{H}, \mathrm{Ar}$ ), 7.33 (ddd, $J=8.0,8.0,1.1 \mathrm{~Hz}, 1 \mathrm{H}, \mathrm{Ar}), 7.48(\mathrm{dd}, J=8.0,8.0 \mathrm{~Hz}$, 1H, Ar), 7.58-7.62 (m, 2H, Ar), 7.64 (dd, $J=8.0,1.1 \mathrm{~Hz}, 1 \mathrm{H}, \mathrm{Ar}$ ), 7.70 (d, $J=8.0 \mathrm{~Hz}, 1 \mathrm{H}, \mathrm{Ar}), 7.97$ (dd, $J=8.0,1.1 \mathrm{~Hz}, 1 \mathrm{H}, \mathrm{Ar}$ ), 10.76 (s, $1 \mathrm{H}, \mathrm{CHO}) ;{ }^{13} \mathrm{C}$ NMR $\left(125 \mathrm{MHz}, \mathrm{CDCl}_{3}\right) \delta 89.3,94.6$, 124.6, 125.8, 126.5, 127.2 (2C), 129.0, 130.1, 132.6, 133.4, 133.5, 133.8, 136.1, 191.9. Anal. Calcd. for $\mathrm{C}_{15} \mathrm{H}_{9} \mathrm{BrO}: \mathrm{C}, 63.18 ; \mathrm{H}$, 3.18. Found: C, 63.20; H, 3.28.

4.2.2. 2-[(2-Bromophenyl)ethynyl]-4-fluorobenzaldehyde (1b). By a procedure identical with that described for the preparation of 1a, 2-ethynyl-4-fluorobenzaldehyde (12b) (100 mg, 0.68 mmol) was converted to $\mathbf{1 b}(169 \mathrm{mg}, 83 \%)$ by the reaction with 1-bromo-2-iodobenzene (13a) $(104 \mu \mathrm{L}, 0.81 \mathrm{mmol}), \mathrm{CuI}(6.4 \mathrm{mg}$, $0.034 \mathrm{mmol}), \mathrm{PdCl}_{2}\left(\mathrm{PPh}_{3}\right)_{2}(23.7 \mathrm{mg}, 0.034 \mathrm{mmol})$, and $\mathrm{Et}_{3} \mathrm{~N}$ $(1.0 \mathrm{~mL})$ in THF $(1.0 \mathrm{~mL})$ at $80{ }^{\circ} \mathrm{C}$ for $1.5 \mathrm{~h}$ : colorless solid: $\mathrm{mp}$ $109{ }^{\circ} \mathrm{C} ;{ }^{1} \mathrm{H}$ NMR $\left(500 \mathrm{MHz}, \mathrm{CDCl}_{3}\right) \delta 7.17$ (ddd, $J=8.0,8.0,2.1$ $\mathrm{Hz}, 1 \mathrm{H}, \mathrm{Ar}$ ), 7.26 (ddd, $J=8.0,8.0,1.7 \mathrm{~Hz}, 1 \mathrm{H}, \mathrm{Ar}$ ), 7.33-7.38 (m, 2H, Ar), $7.60(\mathrm{dd}, J=8.0,1.7 \mathrm{~Hz}, 1 \mathrm{H}, \mathrm{Ar}), 7.65$ (d, $J=8.0$ $\mathrm{Hz}, 1 \mathrm{H}, \mathrm{Ar}), 8.00$ (dd, $J=8.6,5.7 \mathrm{~Hz}, 1 \mathrm{H}, \mathrm{Ar}), 10.67$ (s, $1 \mathrm{H}$, $\mathrm{CHO}) ;{ }^{13} \mathrm{C}$ NMR $\left(125 \mathrm{MHz}, \mathrm{CDCl}_{3}\right) \delta 88.0(\mathrm{~d}, J=2.4 \mathrm{~Hz}), 95.6$, $116.9(\mathrm{~d}, J=21.6 \mathrm{~Hz}), 119.8(\mathrm{~d}, J=22.8 \mathrm{~Hz}), 124.1,125.9$, 127.2, 128.9 (d, $J=10.8 \mathrm{~Hz}), 130.0(\mathrm{~d}, J=9.6 \mathrm{~Hz}), 130.5,132.7$, 132.9, 133.6, $165.6(\mathrm{~d}, J=256.7 \mathrm{~Hz}), 190.2$. Anal. Calcd. for $\mathrm{C}_{15} \mathrm{H}_{8} \mathrm{BrFO}$ : C, 59.43; H, 2.66. Found: C, 59.49; H, 2.80 .

4.2.3. 2-[(2-Bromophenyl)ethynyl]-4-methylbenzaldehyde (1c). By a procedure identical with that described for the preparation of 1a, 2-ethynyl-4-methylbenzaldehyde (12c) (50 mg, 0.35 mmol) was converted to $1 \mathrm{c}(78 \mathrm{mg}, 75 \%)$ by the reaction with 1 bromo-2-iodobenzene (13a) $(53.4 \mu \mathrm{L}, 0.42 \mathrm{mmol}), \mathrm{CuI}(3.3 \mathrm{mg}$, $0.017 \mathrm{mmol}), \mathrm{PdCl}_{2}\left(\mathrm{PPh}_{3}\right)_{2}(12.2 \mathrm{mg}, 0.017 \mathrm{mmol})$, and $\mathrm{Et}_{3} \mathrm{~N}$ $(0.75 \mathrm{~mL})$ in THF $(0.75 \mathrm{~mL})$ at $80{ }^{\circ} \mathrm{C}$ for $1 \mathrm{~h}$ : colorless solid: $\mathrm{mp}$ $79{ }^{\circ} \mathrm{C} ;{ }^{1} \mathrm{H}$ NMR $\left(500 \mathrm{MHz}, \mathrm{CDCl}_{3}\right) \delta 2.43\left(\mathrm{~s}, 3 \mathrm{H}, \mathrm{CCH}_{3}\right) 7.22$ (ddd, $J=8.0,8.0,1.5 \mathrm{~Hz}, 1 \mathrm{H}, \mathrm{Ar}), 7.27$ (d, $J=8.0 \mathrm{~Hz}, 1 \mathrm{H}, \mathrm{Ar}$ ), 7.32 (ddd, $J=8.0,8.0,1.1 \mathrm{~Hz}, 1 \mathrm{H}, \mathrm{Ar}), 7.50$ (s, 1H, Ar), 7.58 (dd, $J=8.0,1.7 \mathrm{~Hz}, 1 \mathrm{H}, \mathrm{Ar}), 7.63$ (dd, $J=8.0,1.1 \mathrm{~Hz}, 1 \mathrm{H}, \mathrm{Ar}), 7.86$ $(\mathrm{d}, J=8.0 \mathrm{~Hz}, 1 \mathrm{H}, \mathrm{Ar}), 10.69$ (s, $1 \mathrm{H}, \mathrm{CHO}) ;{ }^{13} \mathrm{C} \mathrm{NMR}(125 \mathrm{MHz}$, $\left.\mathrm{CDCl}_{3}\right) \delta 21.6,89.5,94.1,124.7,125.8,126.4,127.1,127.2$, 130.0 (2C), 132.6, 133.4, 133.7, 134.0, 144.8, 191.5. Anal. Calcd. for $\mathrm{C}_{16} \mathrm{H}_{11} \mathrm{BrO}$ : $\mathrm{C}, 64.24 \mathrm{H}, 3.71$. Found: $\mathrm{C}, 64.15$; H, 3.82.

4.2.4. 2-[(2-Bromophenyl)ethynyl]-5-fluorobenzaldehyde (1d). By a procedure identical with that described for the preparation of 1a, 2-ethynyl-5-fluorobenzaldehyde (12d) (100 mg, 0.68 mmol) was converted to $1 \mathbf{d}(174 \mathrm{mg}, 85 \%)$ by the reaction with 1-bromo-2-iodobenzene (13a) $(104 \mu \mathrm{L}, 0.81 \mathrm{mmol}), \mathrm{CuI}(6.4 \mathrm{mg}$, $0.034 \mathrm{mmol}), \mathrm{PdCl}_{2}\left(\mathrm{PPh}_{3}\right)_{2}(23.7 \mathrm{mg}, 0.034 \mathrm{mmol})$, and $\mathrm{Et}_{3} \mathrm{~N}$ $(1.0 \mathrm{~mL})$ in THF $(1.0 \mathrm{~mL})$ at $80^{\circ} \mathrm{C}$ for $1.5 \mathrm{~h}$ : colorless solid: $\mathrm{mp}$ $93{ }^{\circ} \mathrm{C} ;{ }^{1} \mathrm{H}$ NMR $\left(500 \mathrm{MHz}, \mathrm{CDCl}_{3}\right) \delta 7.24$ (ddd, $J=7.7,7.7,1.5$ $\mathrm{Hz}, 1 \mathrm{H}, \mathrm{Ar}), 7.28-7.34$ (m, 2H, Ar), 7.58 (dd, $J=7.7,1.4 \mathrm{~Hz}, 1 \mathrm{H}$, 
Ar), 7.62-7.64 (m, 2H, Ar), $7.69(\mathrm{dd}, J=8.6,5.2 \mathrm{~Hz}, 1 \mathrm{H}, \mathrm{Ar})$ $10.70(\mathrm{~d}, J=3.4 \mathrm{~Hz}, 1 \mathrm{H}, \mathrm{CHO}) ;{ }^{13} \mathrm{C}$ NMR $\left(125 \mathrm{MHz}, \mathrm{CDCl}_{3}\right) \delta$ 88.2, 94.4, 113.7 (d, $J=22.8 \mathrm{~Hz}), 121.3(\mathrm{~d}, J=22.8 \mathrm{~Hz}), 122.5$ $(\mathrm{d}, J=3.6 \mathrm{~Hz}), 124.4,125.7,127.2,130.2,132.6,133.4,135.4(\mathrm{~d}$ $J=7.2 \mathrm{~Hz}), 138.1$ (d, $J=7.2 \mathrm{~Hz}), 162.6(\mathrm{~d}, J=253.1 \mathrm{~Hz}), 190.6$. Anal. Calcd. for $\mathrm{C}_{15} \mathrm{H}_{8} \mathrm{BrFO}$ : C, 59.43; H, 2.66. Found: C, 59.60; $\mathrm{H}, 2.92$.

4.2.5. 2-[(2-Bromophenyl)ethynyl]-5-methoxylbenzaldehyde (1e). By a procedure identical with that described for the preparation of 1a, 2-ethynyl-5-methoxybenzaldehyde (12e) (100 mg, 0.62 mmol) was converted to $1 \mathrm{e}(167 \mathrm{mg}, 85 \%)$ by the reaction with 1-bromo-2-iodobenzene (13a) $(95.9 \mu \mathrm{L}, 0.75 \mathrm{mmol}), \mathrm{CuI}(5.9 \mathrm{mg}$, $0.031 \mathrm{mmol}), \mathrm{PdCl}_{2}\left(\mathrm{PPh}_{3}\right)_{2}(21.9 \mathrm{mg}, 0.031 \mathrm{mmol})$, and $\mathrm{Et}_{3} \mathrm{~N}$ $(1.0 \mathrm{~mL})$ in THF $(1.0 \mathrm{~mL})$ at $80^{\circ} \mathrm{C}$ for $1 \mathrm{~h}$ : colorless solid: $\mathrm{mp}$ $102{ }^{\circ} \mathrm{C} ;{ }^{1} \mathrm{H}$ NMR $\left(500 \mathrm{MHz}, \mathrm{CDCl}_{3}\right) \delta 3.88\left(\mathrm{~s}, 3 \mathrm{H}, \mathrm{CH}_{3}\right), 7.14$ (dd, $J=8.6,2.9 \mathrm{~Hz}, 1 \mathrm{H}, \mathrm{Ar}$ ), 7.20 (ddd, $J=7.7,7.7,1.3 \mathrm{~Hz}, 1 \mathrm{H}$, Ar), 7.31 (ddd, $J=7.7,7.7,1.1 \mathrm{~Hz}, 1 \mathrm{H}, \mathrm{Ar}), 7.44(\mathrm{~d}, J=2.9 \mathrm{~Hz}$, $1 \mathrm{H}, \mathrm{Ar}), 7.56$ (dd, $J=8.0,1.7 \mathrm{~Hz}, 1 \mathrm{H}, \mathrm{Ar}), 7.60-7.63(\mathrm{~m}, 2 \mathrm{H}, \mathrm{Ar})$ $10.72(\mathrm{~s}, 1 \mathrm{H}, \mathrm{CHO}) ;{ }^{13} \mathrm{C}$ NMR $\left(125 \mathrm{MHz}, \mathrm{CDCl}_{3}\right) \delta$ 55.6, 89.4, 93.3, 109.8, 119.1, 121.7, 124.9, 125.6, 127.1, 129.7, 132.5, 133.2, 134.7, 137.6, 160.1, 191.8. Anal. Calcd. for $\mathrm{C}_{16} \mathrm{H}_{11} \mathrm{BrO}_{2}$ : C, 60.98 H, 3.52. Found: C, 60.95; H, 3.41 .
$12 f$

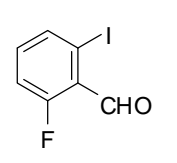

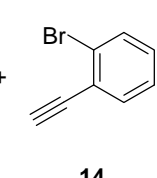

14

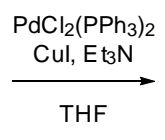

(2)

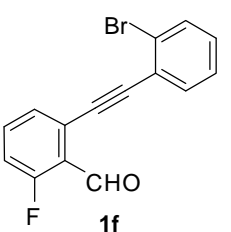

4.2.6. 2-[(2-Bromophenyl)ethynyl]-6-fluorobenzaldehyde (1f). By a procedure similar to that described for the preparation of $\mathbf{1 a}$, 2-fluoro-6-iodobenzaldehyde (12f) $(50 \mathrm{mg}, 0.20 \mathrm{mmol})$ was converted to 1f (56 mg, 92\%) by the reaction with 1-bromo-2ethynybenzene (14) $(43.4 \mathrm{mg}, 0.24 \mathrm{mmol}), \mathrm{CuI}(1.9 \mathrm{mg}, 0.012$ $\mathrm{mmol}), \mathrm{PdCl}_{2}\left(\mathrm{PPh}_{3}\right)_{2}(7.0 \mathrm{mg}, 0.012 \mathrm{mmol})$, and $\mathrm{Et}_{3} \mathrm{~N}(0.5 \mathrm{~mL})$ in THF $(0.5 \mathrm{~mL})$ at $50{ }^{\circ} \mathrm{C}$ for $2 \mathrm{~h}$ : colorless solid: $\mathrm{mp} 74{ }^{\circ} \mathrm{C} ;{ }^{1} \mathrm{H}$ NMR $\left(500 \mathrm{MHz}, \mathrm{CDCl}_{3}\right) \delta 7.16(\mathrm{ddd}, J=7.7,7.7,3.6 \mathrm{~Hz}, 1 \mathrm{H}$, Ar), 7.24 (ddd, $J=7.7,7.7,1.7 \mathrm{~Hz}, 1 \mathrm{H}, \mathrm{Ar}$ ), 7.33 (ddd, $J=7.7$, 7.7, 1.1 Hz, 1H, Ar), 7.50-7.57 (m, 2H, Ar), 7.61-7.64 (m, 2H, Ar), $10.70(\mathrm{~s}, 1 \mathrm{H}, \mathrm{CHO}) ;{ }^{13} \mathrm{C} \mathrm{NMR}\left(125 \mathrm{MHz}, \mathrm{CDCl}_{3}\right) \delta 89.2(\mathrm{~d}$, $J=4.8 \mathrm{~Hz}), 95.2,117.2(\mathrm{~d}, J=21.6 \mathrm{~Hz}), 124.4(\mathrm{~d}, J=9.6 \mathrm{~Hz})$, $125.8,127.0,127.1(\mathrm{~d}, J=25.2 \mathrm{~Hz}), 129.7(2 \mathrm{C}), 130.4,132.6$, 133.7, $134.8(\mathrm{~d}, J=10.8 \mathrm{~Hz}), 162.4(\mathrm{~d}, J=262.7 \mathrm{~Hz}), 188.5$. Anal. Calcd. for $\mathrm{C}_{15} \mathrm{H}_{8} \mathrm{BrFO}$ : C, 59.43; H, 2.66. Found: C, 59.42; H, 2.93 .

4.2.7. 2-[(2-Bromo-5-fluorophenyl)ethynyl]benzaldehyde (1g). By a procedure identical with that described for the preparation of 1a, 2-ethynylbenzaldehyde (12a) $(100 \mathrm{mg}, 0.77 \mathrm{mmol})$ was converted to $1 \mathrm{~g}$ (177 $\mathrm{mg}, 91 \%)$ by the reaction with 1-bromo-4fluoro-2-iodobenzene (13b) $(83.7 \mu \mathrm{L}, 0.64 \mathrm{mmol}), \mathrm{CuI}(6.1 \mathrm{mg}$, $0.032 \mathrm{mmol}), \mathrm{PdCl}_{2}\left(\mathrm{PPh}_{3}\right)_{2}(22.5 \mathrm{mg}, 0.032 \mathrm{mmol})$, and $\mathrm{Et}_{3} \mathrm{~N}$ $(1.5 \mathrm{~mL})$ in THF $(1.5 \mathrm{~mL})$ at $50{ }^{\circ} \mathrm{C}$ for $2 \mathrm{~h}$ : colorless solid: $\mathrm{mp}$ 89-90 ${ }^{\circ} \mathrm{C} ;{ }^{1} \mathrm{H}$ NMR $\left(500 \mathrm{MHz}, \mathrm{CDCl}_{3}\right) \delta 6.96$ (ddd, $J=8.3,8.3$, $3.1 \mathrm{~Hz}, 1 \mathrm{H}, \mathrm{Ar}), 7.29$ (dd, $J=8.3,2.9 \mathrm{~Hz}, 1 \mathrm{H}, \mathrm{Ar}), 7.49$ (dd, $J=$ 7.4, 7.4 Hz, 1H, Ar), 7.55-7.61 (m, 2H, Ar), 7.68 (d, $J=7.4 \mathrm{~Hz}$, $1 \mathrm{H}, \mathrm{Ar}), 7.96$ (dd, $J=7.4,1.1 \mathrm{~Hz}, 1 \mathrm{H}, \mathrm{Ar}), 10.71$ (s, 1H, CHO); ${ }^{13} \mathrm{C}$ NMR $\left(125 \mathrm{MHz}, \mathrm{CDCl}_{3}\right) \delta 90.2,93.4(\mathrm{~d}, J=3.6 \mathrm{~Hz}), 117.7$ $(\mathrm{d}, J=22.8 \mathrm{~Hz}), 112.1(\mathrm{~d}, J=24.0 \mathrm{~Hz}), 120.3(\mathrm{~d}, J=3.6 \mathrm{~Hz})$, $125.7,126.0(\mathrm{~d}, J=9.6 \mathrm{~Hz}), 127.2,129.3,133.4,133.7,133.8(\mathrm{~d}$, $J=9.6 \mathrm{~Hz}), 136.2,161.3(\mathrm{~d}, J=248.3 \mathrm{~Hz}), 191.4$. Anal. Calcd. for $\mathrm{C}_{15} \mathrm{H}_{8} \mathrm{BrFO}$ : C, 59.43; H, 2.66. Found: C, 59.64; H, 2.95.

4.2.8. 2-[(2-Bromo-5-methylphenyl)ethynyl]benzaldehyde (1h). By a procedure identical with that described for the preparation of 1a, 2-ethynylbenzaldehyde (12a) (200 mg, $1.54 \mathrm{mmol})$ was converted to $\mathbf{1 h}$ (331 mg, 72\%) by the reaction with 1-bromo-2iodo-4-methylbenzene (13c) $(182 \mu \mathrm{L}, 1.28 \mathrm{mmol}), \mathrm{CuI}(12.2 \mathrm{mg}$, $0.064 \mathrm{mmol}), \mathrm{PdCl}_{2}\left(\mathrm{PPh}_{3}\right)_{2}(44.9 \mathrm{mg}, 0.064 \mathrm{mmol})$, and $\mathrm{Et}_{3} \mathrm{~N}$ $(3.0 \mathrm{~mL})$ in THF $(3.0 \mathrm{~mL})$ at $80{ }^{\circ} \mathrm{C}$ for $1 \mathrm{~h}$ : colorless solid: $\mathrm{mp}$ 93-94 ${ }^{\circ} \mathrm{C}$; ${ }^{1} \mathrm{H}$ NMR $\left(500 \mathrm{MHz}, \mathrm{CDCl}_{3}\right) \delta 2.33\left(\mathrm{~s}, 3 \mathrm{H}, \mathrm{CCH}_{3}\right)$, 7.04 (dd, $J=8.0,2.0 \mathrm{~Hz}, 1 \mathrm{H}, \mathrm{Ar}), 7.42$ (d, $J=1.7 \mathrm{~Hz}, 1 \mathrm{H}, \mathrm{Ar}$ ), 7.46-7.51 (m, 2H, Ar), 7.60 (ddd, $J=7.4,7.4,1.1 \mathrm{~Hz}, 1 \mathrm{H}, \mathrm{Ar}$ ), 7.69 (d, $J=8.0 \mathrm{~Hz}, 1 \mathrm{H}, \mathrm{Ar}), 7.97$ (dd, $J=8.0,1.1 \mathrm{~Hz}, 1 \mathrm{H}, \mathrm{Ar})$, $10.76(\mathrm{~s}, 1 \mathrm{H}, \mathrm{CHO}) ;{ }^{13} \mathrm{C} \mathrm{NMR}\left(125 \mathrm{MHz}, \mathrm{CDCl}_{3}\right) \delta 20.7,88.9$, 94.9, 122.4, 124.2, 126.6, 127.1, 128.9, 131.2, 132.3, 133.3, 133.7, 134.0, 136.1, 137.2, 192.0. Anal. Calcd. for $\mathrm{C}_{16} \mathrm{H}_{11} \mathrm{BrO}$ : C, 64.24 H, 3.71. Found: C, 64.44; H, 3.88.

4.2.9. 2-(Phenylethynyl)benzaldehyde (1i). By a procedure identical with that described for the preparation of 1a, 2ethynylbenzaldehyde (12a) (100 $\mathrm{mg}, 0.77 \mathrm{mmol}$ ) was converted to $1 \mathbf{i}(111 \mathrm{mg}, 70 \%)$ by the reaction with iodobenzene (13d) (103 $\mu \mathrm{L}, 0.92 \mathrm{mmol}), \mathrm{CuI}(7.3 \mathrm{mg}, 0.038 \mathrm{mmol}), \mathrm{PdCl}_{2}\left(\mathrm{PPh}_{3}\right)_{2}(26.9$ $\mathrm{mg}, 0.038 \mathrm{mmol})$, and $\mathrm{Et}_{3} \mathrm{~N}(1.0 \mathrm{~mL})$ in THF $(1.0 \mathrm{~mL})$ at $80{ }^{\circ} \mathrm{C}$ for $1 \mathrm{~h}$ : yellow oil; ${ }^{1} \mathrm{H}$ NMR $\left(500 \mathrm{MHz}, \mathrm{CDCl}_{3}\right) \delta 7.37-7.39(\mathrm{~m}$, $3 \mathrm{H}, \mathrm{Ar}), 7.44$ (dd, $J=7.7,7.7 \mathrm{~Hz}, 1 \mathrm{H}, \mathrm{Ar}), 7.55-7.59$ (m, 3H, Ar) 7.64 (d, $J=7.4 \mathrm{~Hz}, 1 \mathrm{H}, \mathrm{Ar}), 7.95$ (d, $J=7.4 \mathrm{~Hz}, 1 \mathrm{H}, \mathrm{Ar}), 10.65$ (s, 1H, CHO); ${ }^{13} \mathrm{C}$ NMR $\left(125 \mathrm{MHz}, \mathrm{CDCl}_{3}\right) \delta 84.9,96.3,122.3$, 126.8, 127.2, 128.5 (2C), 128.6, 129.0, 131.7 (2C), 133.2, 133.7, 135.8, 191.6; HRMS (FAB) calcd for $\mathrm{C}_{15} \mathrm{H}_{11} \mathrm{O}\left(\mathrm{MH}^{+}\right)$: 207.0810; found: 207.0810 .

4.2.10. 2-[(4-Methylphenyl)ethynyl]benzaldehyde (1j). Вy a procedure identical with that described for the preparation of 1a, 2-ethynylbenzaldehyde (12a) $(100 \mathrm{mg}, 0.77 \mathrm{mmol})$ was converted to $\mathbf{1 j}$ (120 mg, 71\%) by the reaction with 1-iodo-2methylbenzene (13e) (201 mg, $0.92 \mathrm{mmol}), \mathrm{CuI}$ (7.3 mg, 0.038 $\mathrm{mmol}), \mathrm{PdCl}_{2}\left(\mathrm{PPh}_{3}\right)_{2}(26.9 \mathrm{mg}, 0.038 \mathrm{mmol})$, and $\mathrm{Et}_{3} \mathrm{~N}(1.0 \mathrm{~mL})$ in THF $(1.0 \mathrm{~mL})$ at $80{ }^{\circ} \mathrm{C}$ for $1 \mathrm{~h}$ : colorless solid: $\mathrm{mp} 48{ }^{\circ} \mathrm{C} ;{ }^{1} \mathrm{H}$ NMR (500 MHz, $\left.\mathrm{CDCl}_{3}\right) \delta 2.37\left(\mathrm{~s}, 3 \mathrm{H}, \mathrm{CCH}_{3}\right), 7.18(\mathrm{~d}, J=8.0$ $\mathrm{Hz}, 2 \mathrm{H}, \mathrm{Ar}$ ), 7.40-7.46 (m, 3H, Ar), 7.56 (ddd, $J=7.6,7.6,1.3$ $\mathrm{Hz}, 1 \mathrm{H}, \mathrm{Ar}), 7.62$ (d, $J=6.9 \mathrm{~Hz}, 1 \mathrm{H}, \mathrm{Ar}), 7.93$ (dd, $J=7.6,1.1$ $\mathrm{Hz}, 1 \mathrm{H}, \mathrm{Ar}), 10.65$ (s, 1H, CHO); ${ }^{13} \mathrm{C} \mathrm{NMR}\left(125 \mathrm{MHz}, \mathrm{CDCl}_{3}\right) \delta$ 21.5, 84.3, 96.6, 119.2, 127.1 (2C), 128.3, 129.2 (2C), 131.5 (2C), 133.1, 133.7, 135.7, 139.3, 191.7. Anal. Calcd. for $\mathrm{C}_{16} \mathrm{H}_{12} \mathrm{O}: \mathrm{C}$, 87.25; H, 5.49. Found: C, 87.11; H, 5.74.

\subsection{Copper-catalyzed coupling-cyclization}

4.3.1. General procedure: synthesis of 13-(2-Bromophenyl)7,7a,12,13-dihydroisoquinolino[2,1-a]perimidine (3a) (Table 1, entry 6). A mixture of $\mathbf{1 a}(50 \mathrm{mg}, 0.18 \mathrm{mmol}), 1,8-$ diaminonaphthalene (2) (41.5 mg, $0.26 \mathrm{mmol})$, and $\mathrm{CuI}(3.3 \mathrm{mg}$, $0.018 \mathrm{mmol})$ in dioxane $(1.0 \mathrm{~mL})$ was stirred for $60 \mathrm{~min}$ at $150{ }^{\circ} \mathrm{C}$ under microwave irradiation $(300 \mathrm{~W})$. The reaction mixture was concentrated under reduced pressure and purified by column chromatography over silica gel with hexane-EtOAc $(15: 1)$ to give $3 a(68 \mathrm{mg}, 91 \%)$ as a pale yellow amorphous solid; ${ }^{1} \mathrm{H}$ NMR $\left(500 \mathrm{MHz}, \mathrm{CDCl}_{3}\right) \delta 4.49$ (br s, $\left.1 \mathrm{H}, \mathrm{NH}\right), 5.68(\mathrm{~s}, 1 \mathrm{H}$, $\mathrm{NCHN}), 6.15(\mathrm{~s}, 1 \mathrm{H}, \mathrm{C}=\mathrm{CH}), 6.36(\mathrm{~d}, J=7.4 \mathrm{~Hz}, 1 \mathrm{H}, \mathrm{Ar}), 6.66$ (dd, $J=6.6,2.0 \mathrm{~Hz}, 1 \mathrm{H}, \mathrm{Ar}), 6.96(\mathrm{dd}, J=8.0,8.0 \mathrm{~Hz}, 1 \mathrm{H}, \mathrm{Ar}$ ), 7.04-7.09 (m, 3H, Ar), 7.16 (d, $J=7.4 \mathrm{~Hz}, 1 \mathrm{H}, \mathrm{Ar}), 7.21$ (ddd, $J$ $=7.4,7.4,1.1 \mathrm{~Hz}, 1 \mathrm{H}, \mathrm{Ar}), 7.29-7.36(\mathrm{~m}, 4 \mathrm{H}, \mathrm{Ar}), 7.38-7.40(\mathrm{~m}$, $2 \mathrm{H}, \mathrm{Ar}) ;{ }^{13} \mathrm{C} \mathrm{NMR}\left(125 \mathrm{MHz}, \mathrm{CDCl}_{3}\right) \delta 69.3,103.2,107.6,117.1$, $118.5,120.1,123.0,124.2,124.4,124.9,125.8,126.3,126.4$, 126.6, 126.7, 129.2 (2C), 131.9, 132.2, 132.3, 134.4, 137.8, 138.3, 142.1, 142.4; HRMS (FAB) calcd for $\mathrm{C}_{25} \mathrm{H}_{18} \mathrm{BrN}_{2}\left(\mathrm{MH}^{+}\right)$: 425.0653; found: 425.0649 .

4.3.2.

13-(2-Bromophenyl)-10-fluoro-7,7adihydroisoquinolino[2,1-a]perimidine (3b) (Table 2, entry 1). By a procedure identical with that described for the preparation of $\mathbf{3 a}$, 1b $(50 \mathrm{mg}, 0.17 \mathrm{mmol})$ was converted into $3 \mathbf{b}(70 \mathrm{mg}, 97 \%)$ by 
the reaction with 1,8-diaminonaphthalene (2) (39.1 mg, 0.25 mmol) and $\mathrm{CuI}(3.1 \mathrm{mg}, 0.017 \mathrm{mmol})$ : yellow amorphous solid; ${ }^{1} \mathrm{H}$ NMR $\left(500 \mathrm{MHz}, \mathrm{CDCl}_{3}\right) \delta 4.45$ (br s, $\left.1 \mathrm{H}, \mathrm{NH}\right), 5.57(\mathrm{~s}, 1 \mathrm{H}$, $\mathrm{NCHN}), 6.12(\mathrm{~s}, 1 \mathrm{H}, \mathrm{C}=\mathrm{CH}), 6.32(\mathrm{~d}, J=7.4 \mathrm{~Hz}, 1 \mathrm{H}, \mathrm{Ar}), 6.67$ (dd, $J=6.9,1.7 \mathrm{~Hz}, 1 \mathrm{H}, \mathrm{Ar}), 6.82(\mathrm{dd}, J=9.5,2.6 \mathrm{~Hz}, 1 \mathrm{H}, \mathrm{Ar})$, 6.88 (ddd, $J=8.6,8.6,2.3 \mathrm{~Hz}, 1 \mathrm{H}, \mathrm{Ar}), 6.93$ (dd, $J=8.0,8.0 \mathrm{~Hz}$, $1 \mathrm{H}, \mathrm{Ar}), 7.04-7.10$ (m, 3H, Ar), 7.26-7.32 (m, 3H, Ar), 7.37-7.38 $(\mathrm{m}, 2 \mathrm{H}, \mathrm{Ar}) ;{ }^{13} \mathrm{C}$ NMR $\left(125 \mathrm{MHz}, \mathrm{CDCl}_{3}\right) \delta 69.0,102.2,108.0$, $110.4(\mathrm{~d}, J=21.6 \mathrm{~Hz}), 112.5(\mathrm{~d}, J=22.8 \mathrm{~Hz}), 117.5,118.8$, $120.3,122.1(\mathrm{~d}, J=2.4 \mathrm{~Hz}), 123.5,124.4,124.9,126.5,126.8$, $128.5(\mathrm{~d}, J=9.6 \mathrm{~Hz}), 129.5,131.9,132.4,134.5,134.6(\mathrm{~d}, J=$ $9.6 \mathrm{~Hz}), 137.6,138.0,142.0,143.7,163.5(\mathrm{~d}, J=245.9 \mathrm{~Hz})$; HRMS (FAB) calcd for $\mathrm{C}_{25} \mathrm{H}_{17} \mathrm{BrFN}_{2}\left(\mathrm{MH}^{+}\right)$: 443.0560; found: 443.0558 .

\subsubsection{3-(2-Bromophenyl)-10-methyl-7,7a-} dihydroisoquinolino[2,1-a]perimidine (3c) (Table 2, entry 2). By a procedure identical with that described for the preparation of $\mathbf{3 a}$ 1c $(50 \mathrm{mg}, 0.17 \mathrm{mmol})$ was converted into 3c $(71 \mathrm{mg}, 97 \%)$ by the reaction with 1,8-diaminonaphthalene (2) (39.6 mg, 0.25 $\mathrm{mmol})$ and $\mathrm{CuI}(3.2 \mathrm{mg}, 0.017 \mathrm{mmol})$ : yellow amorphous solid; ${ }^{1} \mathrm{H}$ NMR $\left(500 \mathrm{MHz}, \mathrm{CDCl}_{3}\right) \delta 2.35\left(\mathrm{~s}, 3 \mathrm{H}, \mathrm{CCH}_{3}\right), 4.43$ (br s, $1 \mathrm{H}$, $\mathrm{NH}), 5.59(\mathrm{~s}, 1 \mathrm{H}, \mathrm{NCHN}), 6.10(\mathrm{~s}, 1 \mathrm{H}, \mathrm{C}=\mathrm{CH}), 6.29(\mathrm{~d}, J=7.4$ $\mathrm{Hz}, 1 \mathrm{H}, \mathrm{Ar}$ ), 6.64 (dd, $J=6.9,1.1 \mathrm{~Hz}, 1 \mathrm{H}, \mathrm{Ar}), 6.92$ (dd, $J=7.7$, $7.7 \mathrm{~Hz}, 1 \mathrm{H}, \mathrm{Ar}$ ), 6.95 (br s, 1H, Ar), 7.00-7.07 (m, 4H, Ar), 7.20 $(\mathrm{d}, J=7.4 \mathrm{~Hz}, 1 \mathrm{H}, \mathrm{Ar}), 7.24-7.30$ (m, 2H, Ar), 7.34 (d, $J=8.0$ $\mathrm{Hz}, 1 \mathrm{H}, \mathrm{Ar}), 7.37$ (d, $J=7.4 \mathrm{~Hz}, 1 \mathrm{H}, \mathrm{Ar}) ;{ }^{13} \mathrm{C}$ NMR $(125 \mathrm{MHz}$, $\left.\mathrm{CDCl}_{3}\right) \delta 21.3,69.3,103.2,107.6,117.2,118.5,120.2,123.0$, $123.8,124.5,124.8,124.9,126.4,126.6,126.7$ (2C), 129.2, 132.0, $132.1,132.4,134.5,138.0,138.5,139.0,142.3,142.4$; HRMS (FAB) calcd for $\mathrm{C}_{26} \mathrm{H}_{20} \mathrm{BrN}_{2}\left(\mathrm{MH}^{+}\right)$: 439.0810; found: 439.0805 .

4.3.4.

13-(2-Bromophenyl)-9-fluoro-7,7adihydroisoquinolino[2,1-a]perimidine (3d) (Table 2, entry 3). By a procedure identical with that described for the preparation of $3 \mathbf{a}$, 1d $(50 \mathrm{mg}, 0.17 \mathrm{mmol})$ was converted into $3 d$ (65 $\mathrm{mg}, 89 \%)$ by the reaction with 1,8-diaminonaphthalene (2) $(39.1 \mathrm{mg}, 0.25$ $\mathrm{mmol})$ and $\mathrm{CuI}(3.1 \mathrm{mg}, 0.017 \mathrm{mmol})$ : yellow amorphous solid; ${ }^{1} \mathrm{H}$ NMR $\left(500 \mathrm{MHz}, \mathrm{CDCl}_{3}\right) \delta 4.50($ br s, $1 \mathrm{H}, \mathrm{NH}), 5.67(\mathrm{~s}, 1 \mathrm{H}$, $\mathrm{NCHN}), 6.09(\mathrm{~s}, 1 \mathrm{H}, \mathrm{C}=\mathrm{CH}), 6.31(\mathrm{~d}, J=7.4 \mathrm{~Hz}, 1 \mathrm{H}, \mathrm{Ar}), 6.69$ (dd, $J=6.6,1.4 \mathrm{~Hz}, 1 \mathrm{H}, \mathrm{Ar}), 6.93(\mathrm{dd}, J=7.7,7.7 \mathrm{~Hz}, 1 \mathrm{H}, \mathrm{Ar})$, 7.01-7.12 (m, 6H, Ar), 7.28-7.39 (m, 4H, Ar); ${ }^{13} \mathrm{C}$ NMR $(125$ $\left.\mathrm{MHz}, \mathrm{CDCl}_{3}\right) \delta 69.1(\mathrm{~d}, J=2.4 \mathrm{~Hz}), 103.1,108.2,113.6(\mathrm{~d}, J=$ $22.8 \mathrm{~Hz}), 116.3(\mathrm{~d}, J=21.6 \mathrm{~Hz}), 117.1,118.9,120.1,123.1$, 124.4, 125.0, 126.0 (d, $J=8.4 \mathrm{~Hz}), 126.5,126.8,128.3$ (d, $J=$ $7.2 \mathrm{~Hz}), 128.7(\mathrm{~d}, J=2.4 \mathrm{~Hz}), 129.4,131.9,132.5,134.4,137.6$, 138.1, 141.7, 141.9 (d, $J=2.4 \mathrm{~Hz}), 161.2(\mathrm{~d}, J=244.7 \mathrm{~Hz})$; HRMS (FAB) calcd for $\mathrm{C}_{25} \mathrm{H}_{17} \mathrm{BrFN}_{2}\left(\mathrm{MH}^{+}\right)$: 443.0560; found: 443.0552 .

\subsubsection{3-(2-Bromophenyl)-9-methoxy-7,7a-} dihydroisoquinolino[2,1-a]perimidine (3e) (Table 2, entry 4). By a procedure identical with that described for the preparation of $\mathbf{3 a}$ 1e $(50 \mathrm{mg}, 0.16 \mathrm{mmol})$ was converted into 3 e $(51 \mathrm{mg}, 71 \%)$ by the reaction with 1,8-diaminonaphthalene (2) $(37.6 \mathrm{mg}, 0.24$ $\mathrm{mmol})$ and $\mathrm{CuI}(3.0 \mathrm{mg}, 0.016 \mathrm{mmol})$ : yellow amorphous solid; ${ }^{1} \mathrm{H}$ NMR $\left(500 \mathrm{MHz}, \mathrm{CDCl}_{3}\right) \delta 3.84\left(\mathrm{~s}, 3 \mathrm{H}, \mathrm{OCH}_{3}\right), 4.54$ (br s, $1 \mathrm{H}$ $\mathrm{NH}), 5.68(\mathrm{~s}, 1 \mathrm{H}, \mathrm{NCHN}), 6.09(\mathrm{~s}, 1 \mathrm{H}, \mathrm{C}=\mathrm{CH}), 6.30(\mathrm{~d}, J=7.4$ $\mathrm{Hz}, 1 \mathrm{H}, \mathrm{Ar}), 6.68(\mathrm{dd}, J=6.9,1.1 \mathrm{~Hz}, 1 \mathrm{H}, \mathrm{Ar}), 6.89-6.91(\mathrm{~m}, 2 \mathrm{H}$, Ar), $6.93(\mathrm{dd}, J=8.0,8.0 \mathrm{~Hz}, 1 \mathrm{H}, \mathrm{Ar}), 7.03-7.06(\mathrm{~m}, 1 \mathrm{H}, \mathrm{Ar})$, 7.09-7.11 (m, 3H, Ar), 7.26-7.34 (m, 3H, Ar), 7.39 (d, $J=8.0 \mathrm{~Hz}$ $1 \mathrm{H}, \mathrm{Ar}) ;{ }^{13} \mathrm{C}$ NMR $\left(125 \mathrm{MHz}, \mathrm{CDCl}_{3}\right) \delta 55.5,69.5,103.9,107.8$, $112.2,114.9,116.8,118.6,120.0,122.7,124.6,125.1,125.5$, $125.8,126.4,126.8,128.3,129.2,132.1,132.5,134.5,138.1$, 138.5, 140.5, 142.0, 158.3; HRMS (FAB) calcd for $\mathrm{C}_{26} \mathrm{H}_{20} \mathrm{BrN}_{2} \mathrm{O}$ $\left(\mathrm{MH}^{+}\right)$: 455.0759; found: 455.0756
4.3.6.

13-(2-Bromophenyl)-8-fluoro-7,7adihydroisoquinolino[2,1-a]perimidine (3f) (Table 2, entry 5). By a procedure identical with that described for the preparation of $\mathbf{3 a}$, 1f $(50 \mathrm{mg}, 0.17 \mathrm{mmol})$ was converted into $3 f(65 \mathrm{mg}, 89 \%)$ by the reaction with 1,8-diaminonaphthalene (2) $(39.1 \mathrm{mg}, 0.25$ mmol) and $\mathrm{CuI}(3.1 \mathrm{mg}, 0.017 \mathrm{mmol})$ : yellow amorphous solid; ${ }^{1} \mathrm{H}$ NMR $\left(500 \mathrm{MHz}, \mathrm{CDCl}_{3}\right) \delta 4.53$ (br s, $\left.1 \mathrm{H}, \mathrm{NH}\right), 5.62(\mathrm{~d}, J=$ $1.7 \mathrm{~Hz}, 1 \mathrm{H}, \mathrm{NCHN}), 6.33$ (br s, $1 \mathrm{H}, \mathrm{Ar}), 6.46(\mathrm{~s}, 1 \mathrm{H}, \mathrm{C}=\mathrm{CH})$, 6.70 (dd, $J=6.9,1.7 \mathrm{~Hz}, 1 \mathrm{H}, \mathrm{Ar}), 6.89-6.95$ (m, 3H, Ar), 7.057.08 (m, 2H, Ar), 7.27-7.33 (m, 3H, Ar), 7.38-7.40 (m, 2H, Ar); ${ }^{13} \mathrm{C}$ NMR $\left(125 \mathrm{MHz}, \mathrm{CDCl}_{3}\right) \delta 64.3,102.2,107.8,112.2(\mathrm{~d}, J=$ $21.6 \mathrm{~Hz}), 113.0(\mathrm{~d}, J=15.6 \mathrm{~Hz}), 117.6,118.7,120.0(\mathrm{~d}, J=2.4$ $\mathrm{Hz}), 123.6,124.4,124.8,126.5,126.8,129.4,130.6$ (d, $J=9.6$ $\mathrm{Hz}), 131.8,132.4,134.4,134.6(\mathrm{~d}, J=4.8 \mathrm{~Hz}), 137.5,138.1$, 140.9, 142.6, 143.5, 159.9 (d, $J=245.9 \mathrm{~Hz})$; HRMS (FAB) calcd for $\mathrm{C}_{25} \mathrm{H}_{17} \mathrm{BrFN}_{2}\left(\mathrm{MH}^{+}\right)$: 443.0560 ; found: 443.0555

4.3.7.

13-(2-Bromo-5-fluorophenyl)-7,7adihydroisoquinolino[2,1-a]perimidine (3g) (Table 2, entry 6). By a procedure identical with that described for the preparation of $\mathbf{3 a}$ $1 \mathrm{~g}(50 \mathrm{mg}, 0.17 \mathrm{mmol})$ was converted into $3 \mathrm{~g}(53 \mathrm{mg}, 73 \%)$ by the reaction with 1,8-diaminonaphthalene (2) (39.1 mg, 0.25 $\mathrm{mmol})$ and $\mathrm{CuI}(3.1 \mathrm{mg}, 0.017 \mathrm{mmol})$ : pale yellow amorphous solid; ${ }^{1} \mathrm{H}$ NMR $\left(500 \mathrm{MHz}, \mathrm{CDCl}_{3}\right) \delta 4.48($ br s, $1 \mathrm{H}, \mathrm{NH}), 5.64(\mathrm{~s}$, $1 \mathrm{H}, \mathrm{NCHN}), 6.12(\mathrm{~s}, 1 \mathrm{H}, \mathrm{C}=\mathrm{CH}), 6.33(\mathrm{~d}, J=7.4 \mathrm{~Hz}, 1 \mathrm{H}, \mathrm{Ar})$, 6.66 (dd, $J=6.3,1.7 \mathrm{~Hz}, 1 \mathrm{H}, \mathrm{Ar}$ ), 6.79 (ddd, $J=8.4,8.4,3.2 \mathrm{~Hz}$, $1 \mathrm{H}, \mathrm{Ar}), 6.89$ (br s, 1H, Ar), 6.97 (dd, $J=7.7,7.7 \mathrm{~Hz}, 1 \mathrm{H}, \mathrm{Ar}$ ), $7.13(\mathrm{~d}, J=7.4 \mathrm{~Hz}, 1 \mathrm{H}, \mathrm{Ar}), 7.21(\mathrm{ddd}, J=7.4,7.4,1.1 \mathrm{~Hz}, 1 \mathrm{H}$, Ar), 7.27-7.34 (m, 5H, Ar), $7.38(\mathrm{~d}, J=7.4 \mathrm{~Hz}, 1 \mathrm{H}, \mathrm{Ar}) ;{ }^{13} \mathrm{C}$ NMR $\left(125 \mathrm{MHz}, \mathrm{CDCl}_{3}\right) \delta 69.3,103.5,107.9,116.6(\mathrm{~d}, J=22.8$ $\mathrm{Hz}), 116.9,118.7,118.9$ (d, $J=3.6 \mathrm{~Hz}), 119.0(\mathrm{~d}, J=22.8 \mathrm{~Hz})$, $120.2,123.3,124.5,124.9,126.2,126.5,126.6,126.7,129.3$, $132.0,133.7$ (d, $J=7.2 \mathrm{~Hz}), 134.5,137.6,140.1(\mathrm{~d}, J=8.4 \mathrm{~Hz})$, 141.5, 142.0, 161.3 (d, $J=248.3 \mathrm{~Hz}$ ); HRMS (FAB) calcd for $\mathrm{C}_{25} \mathrm{H}_{17} \mathrm{BrFN}_{2}\left(\mathrm{MH}^{+}\right)$: 443.0560; found: 443.0560 .

4.3.8.

13-(2-Bromo-5-methylphenyl)-7,7adihydroisoquinolino[2,1-a]perimidine (3h) (Table 2, entry 7). By a procedure identical with that described for the preparation of $\mathbf{3 a}$, 1h $(50 \mathrm{mg}, 0.17 \mathrm{mmol})$ was converted into $3 \mathbf{h}(67 \mathrm{mg}, 91 \%)$ by the reaction with 1,8-diaminonaphthalene (2) (39.6 mg, 0.25 mmol) and $\mathrm{CuI}(3.2 \mathrm{mg}, 0.017 \mathrm{mmol})$ : yellow amorphous solid; ${ }^{1} \mathrm{H}$ NMR $\left(500 \mathrm{MHz}, \mathrm{CDCl}_{3}\right) \delta 2.16\left(\mathrm{~s}, 3 \mathrm{H}, \mathrm{CCH}_{3}\right), 4.52($ br s, $1 \mathrm{H}$, $\mathrm{NH}), 5.64(\mathrm{~s}, 1 \mathrm{H}, \mathrm{NCHN}), 6.15(\mathrm{~s}, 1 \mathrm{H}, \mathrm{C}=\mathrm{CH}), 6.31(\mathrm{~d}, J=7.4$ $\mathrm{Hz}, 1 \mathrm{H}, \mathrm{Ar}$ ), 6.67 (dd, $J=6.6,1.4 \mathrm{~Hz}, 1 \mathrm{H}, \mathrm{Ar}$ ), 6.87 (dd, $J=8.3$, $2.0 \mathrm{~Hz}, 1 \mathrm{H}, \mathrm{Ar}), 6.95$ (dd, $J=8.0,8.0 \mathrm{~Hz}, 1 \mathrm{H}, \mathrm{Ar}), 6.98$ (br s, $1 \mathrm{H}$ Ar), 7.13 (d, $J=7.4 \mathrm{~Hz}, 1 \mathrm{H}, \mathrm{Ar}), 7.18-7.22$ (m, 2H, Ar), 7.26$7.33(\mathrm{~m}, 4 \mathrm{H}, \mathrm{Ar}), 7.36(\mathrm{~d}, J=8.0 \mathrm{~Hz}, 1 \mathrm{H}, \mathrm{Ar}) ;{ }^{13} \mathrm{C}$ NMR $(125$ $\left.\mathrm{MHz}, \mathrm{CDCl}_{3}\right) \delta 20.7,69.4,103.2,107.8,117.0,118.6,120.3$, $121.0,123.0,124.3,124.9,125.8,126.4,126.5,126.6,129.3$, $130.2,132.1,132.4,132.6,134.5,136.7,137.9,138.0,142.2$, 142.7; HRMS (FAB) calcd for $\mathrm{C}_{26} \mathrm{H}_{20} \mathrm{BrN}_{2}\left(\mathrm{MH}^{+}\right)$: 439.0810 ; found: 439.0807

4.3.9. 13-Phenyl-7,7a-dihydroisoquinolino[2,1-a]perimidine (3i) (Table 2, entry 8). By a procedure identical with that described for the preparation of $3 \mathbf{3 a}, \mathbf{1 i}(50 \mathrm{mg}, 0.24 \mathrm{mmol})$ was converted into $3 \mathbf{i}(76 \mathrm{mg}, 91 \%)$ by the reaction with 1,8 diaminonaphthalene (2) $(57.5 \mathrm{mg}, 0.36 \mathrm{mmol})$ and $\mathrm{CuI}(4.6 \mathrm{mg}$, $0.024 \mathrm{mmol})$ : pale yellow amorphous solid; ${ }^{1} \mathrm{H}$ NMR $(500 \mathrm{MHz}$, $\left.\mathrm{CDCl}_{3}\right) \delta 4.94($ br s, $1 \mathrm{H}, \mathrm{NH}), 5.80(\mathrm{~s}, 1 \mathrm{H}, \mathrm{NCHN}), 6.14(\mathrm{~d}, J=$ $7.4 \mathrm{~Hz}, 1 \mathrm{H}, \mathrm{Ar}), 6.55$ (br s, $1 \mathrm{H}, \mathrm{C}=\mathrm{CH}), 6.77(\mathrm{~d}, J=7.4 \mathrm{~Hz}, 1 \mathrm{H}$, Ar), $6.92(\mathrm{dd}, J=7.7,7.7 \mathrm{~Hz}, 1 \mathrm{H}, \mathrm{Ar}), 7.14(\mathrm{dd}, J=7.4,7.4 \mathrm{~Hz}$, 1H, Ar), 7.18-7.22 (m, 4H, Ar), 7.27-7.32 (m, 5H, Ar), 7.49 (m, $2 \mathrm{H}, \mathrm{Ar}) ;{ }^{13} \mathrm{C}$ NMR $\left(125 \mathrm{MHz}, \mathrm{CDCl}_{3}\right) \delta 68.3,107.5,113.2,116.7$, $118.2,120.3$ (2C), 124.4 (2C), 125.1, 125.9, 126.6, 126.9, 127.1 (2C), 128.3 (2C), 128.4 (2C), 133.5, 134.3, 136.6, 138.1, 139.7, 
144.5; HRMS (FAB) calcd for $\mathrm{C}_{25} \mathrm{H}_{19} \mathrm{~N}_{2}\left(\mathrm{MH}^{+}\right)$: 347.1548; found: 347.1547 .

4.3.10. 13-(p-Tolyl)-7,7a-dihydroisoquinolino[2,1-a]perimidine (3j) (Table 2, entry 9). By a procedure identical with that described for the preparation of $\mathbf{3 a}, \mathbf{1} \mathbf{j}(50 \mathrm{mg}, 0.23 \mathrm{mmol})$ was converted into $3 \mathbf{j}(75 \mathrm{mg}, 91 \%)$ by the reaction with 1,8 diaminonaphthalene (2) $(53.8 \mathrm{mg}, 0.34 \mathrm{mmol})$ and $\mathrm{CuI}(4.3 \mathrm{mg}$, $0.023 \mathrm{mmol})$ : yellow amorphous solid; ${ }^{1} \mathrm{H}$ NMR $(500 \mathrm{MHz}$ $\left.\mathrm{CDCl}_{3}\right) \delta 2.33\left(\mathrm{~s}, 3 \mathrm{H}, \mathrm{CCH}_{3}\right), 4.97$ (br s, 1H, NH), $5.76(\mathrm{~s}, 1 \mathrm{H}$, $\mathrm{NCHN}), 6.15(\mathrm{~d}, J=7.4 \mathrm{~Hz}, 1 \mathrm{H}, \mathrm{Ar}), 6.58(\mathrm{br} \mathrm{s}, 1 \mathrm{H}, \mathrm{C}=\mathrm{CH})$, 6.77 (d, $J=7.4 \mathrm{~Hz}, 1 \mathrm{H}, \mathrm{Ar}), 6.93(\mathrm{dd}, J=8.0,8.0 \mathrm{~Hz}, 1 \mathrm{H}, \mathrm{Ar})$, 7.08-7.14 (m, 3H, Ar), 7.17-7.21 (m, 4H, Ar), 7.27 (d, J=7.4 Hz $1 \mathrm{H}, \mathrm{Ar}$ ), 7.30 (dd, $J=7.7,7.7 \mathrm{~Hz}, 1 \mathrm{H}, \mathrm{Ar}), 7.43$ (m, $2 \mathrm{H}, \mathrm{Ar}) ;{ }^{13} \mathrm{C}$ NMR $\left(125 \mathrm{MHz}, \mathrm{CDCl}_{3}\right) \delta 21.3,68.2,107.5,112.8,116.4,118.1$ 120.0 (2C), 124.2 (2C), 125.0, 126.0, 126.6, 126.8, 126.9 (2C), $128.2,129.2(2 \mathrm{C}), 132.3,133.8,134.2,138.2,138.3,139.6$, 144.7; HRMS (FAB) calcd for $\mathrm{C}_{26} \mathrm{H}_{21} \mathrm{~N}_{2}\left(\mathrm{MH}^{+}\right)$: 361.1705; found: 361.1705 .

4.3.11. General procedure for four-component couplingcyclization: synthesis of $N$-[(7,7a-dihydroisoquinolino[2,1a]perimidin-13-yl)methyl]- $N$-isopropylpropan-2-amine (11a) (Table 3, entry 1). A mixture of 2-ethynylbenzaldehyde (8) (30 $\mathrm{mg}, 0.23 \mathrm{mmol})$, paraformaldehyde (9) $(13.8 \mathrm{mg}, 0.46 \mathrm{mmol})$, diisopropylamine (10a) $(65.3 \mu \mathrm{L}, 0.46 \mathrm{mmol})$, and $\mathrm{CuI}(4.4 \mathrm{mg}$, $0.023 \mathrm{mmol})$ in dioxane $(1.0 \mathrm{~mL})$ was stirred at $\mathrm{rt}$ for $1 \mathrm{~h}$. After the Mannich-type reaction was completed (monitored by TLC), 1,8-diaminonaphthalene (2) (109 mg, $0.69 \mathrm{mmol})$ was added, and the mixture was stirred for additional $60 \mathrm{~min}$ at $150{ }^{\circ} \mathrm{C}$ under microwave irradiation $(300 \mathrm{~W})$. The mixture was concentrated under reduced pressure and purified by column chromatography over silica gel with hexane-EtOAc $(25: 1)$ to give 11a (46 mg, $52 \%$ yield) as a pale yellow amorphous solid; ${ }^{1} \mathrm{H}$ NMR $(500$ $\left.\mathrm{MHz}, \mathrm{CDCl}_{3}\right) \delta 0.68\left(\mathrm{~d}, J=6.3 \mathrm{~Hz}, 6 \mathrm{H}, 2 \times \mathrm{CCH}_{3}\right), 0.87(\mathrm{~d}, J=$ $\left.6.3 \mathrm{~Hz}, 6 \mathrm{H}, 2 \times \mathrm{CCH}_{3}\right), 2.88-2.97(\mathrm{~m}, 3 \mathrm{H}, 2 \times \mathrm{N}-\mathrm{CH}$ and $\mathrm{N}-$ $\mathrm{CHH}), 3.37(\mathrm{~d}, J=16.0 \mathrm{~Hz}, 1 \mathrm{H}, \mathrm{N}-\mathrm{CHH}), 4.25$ (br s, $1 \mathrm{H}, \mathrm{NH})$, $5.87(\mathrm{~s}, 1 \mathrm{H}, \mathrm{NCHN}), 6.10(\mathrm{~s}, 1 \mathrm{H}, \mathrm{C}=\mathrm{CH}), 6.58(\mathrm{dd}, J=5.7,2.3$ $\mathrm{Hz}, 1 \mathrm{H}, \mathrm{Ar}), 7.09-7.13(\mathrm{~m}, 3 \mathrm{H}, \mathrm{Ar}), 7.21(\mathrm{~d}, J=6.9 \mathrm{~Hz}, 1 \mathrm{H}, \mathrm{Ar})$, 7.27-7.31 (m, 3H, Ar), 7.36 (dd, $J=7.7,7.7 \mathrm{~Hz}, 1 \mathrm{H}, \mathrm{Ar}), 7.57$ (d, $J=8.6 \mathrm{~Hz}, 1 \mathrm{H}, \mathrm{Ar}) ;{ }^{13} \mathrm{C}$ NMR $\left(125 \mathrm{MHz}, \mathrm{CDCl}_{3}\right) \delta 20.4(2 \mathrm{C})$, 20.5 (2C), 47.5, 48.0 (2C), 70.1, 101.4, 107.3, 118.0, 118.1, $120.0,123.7,124.0,124.9,125.5,126.4$ (2C), 126.6, 129.2, 133.2 134.7, 138.6, 142.8, 144.0; HRMS (FAB) calcd for $\mathrm{C}_{26} \mathrm{H}_{30} \mathrm{~N}_{3}$ $\left(\mathrm{MH}^{+}\right)$: 384.2440 ; found: 384.2438

4.3.12. 13-(Piperidin-1-ylmethyl)-7,7a-dihydroisoquinolino[2,1a]perimidine (11b) (Table 3, entry 2). By a procedure identical with that described for the preparation of 11a, $8(30 \mathrm{mg}, 0.23$ mmol) was converted into $11 \mathbf{b}(60 \mathrm{mg}, 70 \%)$ by the reaction with piperidine (10b) $(45.6 \mu \mathrm{L}, 0.46 \mathrm{mmol})$ : pale yellow amorphous solid; ${ }^{1} \mathrm{H}$ NMR (500 MHz, $\left.\mathrm{CDCl}_{3}\right) \delta 1.00-1.04\left(\mathrm{~m}, 4 \mathrm{H}, 2 \times \mathrm{CH}_{2}\right.$ ), 1.13 (br t, $\left.J=5.4 \mathrm{~Hz}, 2 \mathrm{H}, \mathrm{CH}_{2}\right), 2.03-2.16\left(\mathrm{~m}, 4 \mathrm{H}, 2 \times \mathrm{NCH}_{2}\right.$ ), $2.73(\mathrm{~d}, J=13.2 \mathrm{~Hz}, 1 \mathrm{H}, \mathrm{NCHH}), 3.10(\mathrm{~d}, J=13.2 \mathrm{~Hz}, 1 \mathrm{H}, \mathrm{N}-$ $\mathrm{CHH}), 4.17$ (br s, 1H, NH), $5.72(\mathrm{~s}, 1 \mathrm{H}, \mathrm{NCHN}), 5.88(\mathrm{~s}, 1 \mathrm{H}$, $\mathrm{C}=\mathrm{CH}), 6.54(\mathrm{dd}, J=6.9,1.1 \mathrm{~Hz}, 1 \mathrm{H}, \mathrm{Ar}), 7.09(\mathrm{~d}, J=7.4 \mathrm{~Hz}$, $1 \mathrm{H}, \mathrm{Ar}), 7.13$ (ddd, $J=7.4,7.4,1.1 \mathrm{~Hz}, 1 \mathrm{H}, \mathrm{Ar}), 7.19$ (d, $J=6.9$ $\mathrm{Hz}, 1 \mathrm{H}, \mathrm{Ar}$ ), 7.22-7.35 (m, 5H, Ar), 7.55 (dd, $J=7.7,2.0 \mathrm{~Hz}, 1 \mathrm{H}$, $\mathrm{Ar}) ;{ }^{13} \mathrm{C}$ NMR $\left(125 \mathrm{MHz}, \mathrm{CDCl}_{3}\right) \delta 24.3,25.7$ (2C), 53.5 (2C), $60.8,69.7,103.1,107.1,117.6,118.1,120.5,123.7,124.0,125.4$ (2C), 126.3, 126.5, 126.7, 129.2, 132.6, 134.6, 139.5, 141.2, 142.6; HRMS (FAB) calcd for $\mathrm{C}_{25} \mathrm{H}_{26} \mathrm{~N}_{3}\left(\mathrm{MH}^{+}\right)$: 368.2127; found: 368.2130 .

4.3.13. 4-[(7,7a-Dihydroisoquinolino[2,1-a]perimidin-13yl)methyl]morpholine (11c) (Table 3, entry 3). By a procedure identical with that described for the preparation of 11a, 8 (30 mg, $0.23 \mathrm{mmol})$ was converted into $11 \mathrm{c}(52 \mathrm{mg}, 61 \%)$ by the reaction with morpholine (10c) $(40.2 \mu \mathrm{L}, 0.46 \mathrm{mmol})$ : yellow amorphous solid; ${ }^{1} \mathrm{H}$ NMR $\left(500 \mathrm{MHz}, \mathrm{CDCl}_{3}\right) \delta 1.95$ (br m, $2 \mathrm{H}$, $2 \times \mathrm{N}-\mathrm{CHH}), 2.25$ (br m, 2H, 2× N-CHH), $2.72(\mathrm{~d}, J=13.2 \mathrm{~Hz}$, $1 \mathrm{H}, \mathrm{N}-\mathrm{CHH}), 2.93-3.00\left(\mathrm{~m}, 4 \mathrm{H}, 2 \times \mathrm{OCH}_{2}\right), 3.33(\mathrm{~d}, J=13.2 \mathrm{~Hz}$, $1 \mathrm{H}, \mathrm{N}-\mathrm{CHH}), 4.19$ (br s, $1 \mathrm{H}, \mathrm{NH}), 5.72(\mathrm{~s}, 1 \mathrm{H}, \mathrm{NCHN}), 5.90(\mathrm{~s}$, $1 \mathrm{H}, \mathrm{C}=\mathrm{CH}), 6.56(\mathrm{~d}, J=6.9 \mathrm{~Hz}, 1 \mathrm{H}, \mathrm{Ar}), 7.11(\mathrm{~d}, J=7.4 \mathrm{~Hz}, 1 \mathrm{H}$, Ar), 7.17 (dd, $J=7.2,7.2 \mathrm{~Hz}, 1 \mathrm{H}, \mathrm{Ar}), 7.21-7.35$ (m, 6H, Ar), $7.57(\mathrm{~d}, J=8.6 \mathrm{~Hz}, 1 \mathrm{H}, \mathrm{Ar}) ;{ }^{13} \mathrm{C}$ NMR $\left(125 \mathrm{MHz}, \mathrm{CDCl}_{3}\right) \delta 52.3$ (2C), 60.2, 66.6 (2C), 69.7, 104.0, 107.1, 117.1, 118.1, 120.8, 123.9, 124.2, 125.3, 125.7, 126.6 (2C), 127.0, 129.4, 132.4, 134.7, 139.8, 140.4, 142.5; HRMS (FAB) calcd for $\mathrm{C}_{24} \mathrm{H}_{24} \mathrm{~N}_{3} \mathrm{O}\left(\mathrm{MH}^{+}\right)$: 370.1919; found: 370.1921 .

\subsection{Palladium-catalyzed $\mathrm{C}-\mathrm{H}$ arylation}

4.4.1. General procedure: synthesis of dibenzo[1,2:7,8]quinolizino[3,4,5,6-kla]perimidine (4a) hydrochloride (Table 4, entry 15). A mixture of 3a (50 mg, 0.12 $\mathrm{mmol}), \mathrm{Pd}(\mathrm{OAc})_{2}(2.7 \mathrm{mg}, 0.012 \mathrm{mmol}), \mathrm{PPh}_{3}(7.7 \mathrm{mg}, 0.029$ $\mathrm{mmol}$ ), and $\mathrm{K}_{3} \mathrm{PO}_{4}(50.2 \mathrm{mg}, 0.24 \mathrm{mmol})$ in DMF $(1.5 \mathrm{~mL})$ was stirred for $4 \mathrm{~h}$ at $130{ }^{\circ} \mathrm{C}$. The reaction mixture was concentrated under reduced pressure and purified by column chromatography over silica gel with toluene-EtOAc (200:1) to give 4a (32 mg, $78 \%$ ) as a red solid. When the $\mathrm{C}-\mathrm{H}$ arylation products were poorly soluble in various organic solvents, their hydrochlorides were prepared as follows: the solid was dissolved in $\mathrm{CHCl}_{3}$, and $4 \mathrm{~N}$ solution of $\mathrm{HCl}$ in dioxane was added to the mixture. The precipitates were collected by filtration to give $\mathbf{4 a} \cdot \mathrm{HCl}$ as a brown solid: $\mathrm{mp}$ 124-126 ${ }^{\circ} \mathrm{C}$; ${ }^{1} \mathrm{H}$ NMR $\left(500 \mathrm{MHz}, \mathrm{CD}_{3} \mathrm{OD}\right.$, $\left.60{ }^{\circ} \mathrm{C}\right) \delta 6.58(\mathrm{~d}, J=7.4 \mathrm{~Hz}, 1 \mathrm{H}, \mathrm{Ar}), 6.69(\mathrm{~d}, J=6.9 \mathrm{~Hz}, 1 \mathrm{H}$, Ar), 6.74-6.79 (m, 2H, Ar), 6.93 (br s, 2H, Ar), 7.19 (d, $J=8.0$ $\mathrm{Hz}, 1 \mathrm{H}, \mathrm{Ar}), 7.25$ (br s, 1H, Ar), 7.44-7.50 (m, 3H, Ar), 7.58 (br s, $1 \mathrm{H}, \mathrm{Ar}), 7.67$ (dd, $J=6.6,6.6 \mathrm{~Hz}, 1 \mathrm{H}, \mathrm{Ar}), 8.18(\mathrm{~d}, J=8.0 \mathrm{~Hz}$, $1 \mathrm{H}, \mathrm{Ar}) ;{ }^{13} \mathrm{C}$ NMR $\left(125 \mathrm{MHz}, \mathrm{CD}_{3} \mathrm{OD}, 60{ }^{\circ} \mathrm{C}\right) \delta 110.9,111.2$, $116.1,116.9,119.7,122.5,123.4,123.8,124.7,125.6,125.7$, $126.8,128.1,128.4,129.2,131.0,131.2,131.3,133.0(2 \mathrm{C}), 134.8$ 134.9, 137.1, 137.2, 148.9; HRMS (FAB) calcd for $\mathrm{C}_{25} \mathrm{H}_{15} \mathrm{~N}_{2}$ $\left(\mathrm{MH}^{+}\right)$: 343.1235; found: 343.1233

4.4.2. 12-Fluorodibenzo[1,2:7,8]quinolizino[3,4,5,6kla]perimidine (4b) (Table 5, entry 1). A mixture of $\mathbf{3 b}(30 \mathrm{mg}$, $0.068 \mathrm{mmol}), \mathrm{Pd}(\mathrm{OAc})_{2}(1.5 \mathrm{mg}, 0.007 \mathrm{mmol}), \mathrm{PPh}_{3}(4.4 \mathrm{mg}$, $0.017 \mathrm{mmol})$, and $\mathrm{K}_{3} \mathrm{PO}_{4}(28.7 \mathrm{mg}, 0.14 \mathrm{mmol})$ in DMF $(1.0 \mathrm{~mL})$ was stirred for $4 \mathrm{~h}$ at $130{ }^{\circ} \mathrm{C}$. The reaction mixture was concentrated under reduced pressure and purified by column chromatography over silica gel with toluene-EtOAc (200:1) to give $\mathbf{4 b}$ including some impurities. Recrystallization from pyridine gave pure $\mathbf{4 b}(11 \mathrm{mg}, 45 \%)$ as red crystals: $\mathrm{mp} 298$ $300{ }^{\circ} \mathrm{C} ;{ }^{1} \mathrm{H}$ NMR $\left(500 \mathrm{MHz}, \mathrm{CDCl}_{3}, 60{ }^{\circ} \mathrm{C}\right) \delta 6.57$ (s, $\left.1 \mathrm{H}, \mathrm{Ar}\right)$, $6.63(\mathrm{~d}, J=7.4 \mathrm{~Hz}, 1 \mathrm{H}, \mathrm{Ar}), 6.79$ (dd, $J=4.6,4.6 \mathrm{~Hz}, 1 \mathrm{H}, \mathrm{Ar})$, 6.90 (dd, $J=8.3,8.3 \mathrm{~Hz}, 2 \mathrm{H}, \mathrm{Ar}), 7.09-7.14$ (m, 2H, Ar), 7.22$7.28(\mathrm{~m}, 1 \mathrm{H}, \mathrm{Ar}), 7.36(\mathrm{dd}, J=7.4,7.4 \mathrm{~Hz}, 1 \mathrm{H}, \mathrm{Ar}), 7.56(\mathrm{~d}, J=$ $9.2 \mathrm{~Hz}, 1 \mathrm{H}, \mathrm{Ar}), 7.70(\mathrm{~d}, J=8.0 \mathrm{~Hz}, 1 \mathrm{H}, \mathrm{Ar}), 7.75(\mathrm{~d}, J=8.0 \mathrm{~Hz}$, $1 \mathrm{H}, \mathrm{Ar}), 8.33$ (dd, $J=8.9,6.0 \mathrm{~Hz}, 1 \mathrm{H}, \mathrm{Ar}) ;{ }^{13} \mathrm{C} \mathrm{NMR}$ assignment was difficult due to the poor solubility of $\mathbf{4 b}$ as well as $\mathrm{C}-\mathrm{F}$ couplings; HRMS (FAB) calcd for $\mathrm{C}_{25} \mathrm{H}_{14} \mathrm{FN}_{2}\left(\mathrm{MH}^{+}\right)$: 361.1141 ; found: 361.1143 .

4.4.3. 12-Methyldibenzo[1,2:7,8]quinolizino[3,4,5,6kla]perimidine (4c) hydrochloride (Table 5, entry 2). Вy a procedure identical with that described for the preparation of $\mathbf{4 b}$, 3c $(30 \mathrm{mg}, 0.068 \mathrm{mmol})$ was converted into $4 \mathrm{c}(13 \mathrm{mg}, 53 \%)$ by the reaction with $\mathrm{Pd}(\mathrm{OAc})_{2}(1.5 \mathrm{mg}, 0.007 \mathrm{mmol}), \mathrm{PPh}_{3}(4.5 \mathrm{mg}$, $0.017 \mathrm{mmol})$, and $\mathrm{K}_{3} \mathrm{PO}_{4}(29.0 \mathrm{mg}, 0.14 \mathrm{mmol})$ as red crystals. The crystals were dissolved in $\mathrm{CHCl}_{3}$, and $4 \mathrm{~N}$ solution of $\mathrm{HCl}$ in dioxane was added to the mixture. After hexane was added to the mixture, the precipitates were collected by filtration to give 4c. $\mathrm{HCl}$ as a brown solid: mp $128-130{ }^{\circ} \mathrm{C} ;{ }^{1} \mathrm{H}$ NMR $(500 \mathrm{MHz}$, 


\section{Tetrahedron}

$\left.\mathrm{CD}_{3} \mathrm{OD}\right) \delta 2.39\left(\mathrm{~s}, 3 \mathrm{H}, \mathrm{CCH}_{3}\right), 6.61(\mathrm{~d}, J=5.7 \mathrm{~Hz}, 1 \mathrm{H}, \mathrm{Ar})$ $6.73(\mathrm{~d}, J=8.0 \mathrm{~Hz}, 1 \mathrm{H}, \mathrm{Ar}), 6.88(\mathrm{dd}, J=7.4,7.4 \mathrm{~Hz}, 1 \mathrm{H}, \mathrm{Ar})$, $6.96(\mathrm{~d}, J=9.2 \mathrm{~Hz}, 1 \mathrm{H}, \mathrm{Ar}), 7.00(\mathrm{dd}, J=7.4,7.4 \mathrm{~Hz}, 1 \mathrm{H}, \mathrm{Ar})$, 7.07 (dd, $J=7.4,7.4 \mathrm{~Hz}, 1 \mathrm{H}, \mathrm{Ar}), 7.17$ (s, 1H, Ar), 7.29 (d, $J=$ $8.0 \mathrm{~Hz}, 1 \mathrm{H}, \mathrm{Ar}), 7.39$ (d, $J=8.6 \mathrm{~Hz}, 1 \mathrm{H}, \mathrm{Ar}), 7.43-7.45$ (m, $2 \mathrm{H}$, Ar), $7.60(\mathrm{~d}, J=7.4 \mathrm{~Hz}, 1 \mathrm{H}, \mathrm{Ar}), 7.97(\mathrm{~d}, J=8.0 \mathrm{~Hz}, 1 \mathrm{H}, \mathrm{Ar})$; ${ }^{13} \mathrm{C}$ NMR $\left(125 \mathrm{MHz}, \mathrm{CD}_{3} \mathrm{OD}\right) \delta 22.1,110.3,110.9,113.9,116.9$, $119.5,122.6,123.5,123.7,124.7,125.4,125.5,126.9,128.0$, $128.3,128.4,130.9,131.2,132.9,133.0,133.1,134.9,135.0$, 137.1, 148.5, 149.3; HRMS (FAB) calcd for $\mathrm{C}_{26} \mathrm{H}_{17} \mathrm{~N}_{2}\left(\mathrm{MH}^{+}\right)$: 357.1392; found: 357.1390 .

\subsubsection{3-Fluorodibenzo[1,2:7,8]quinolizino[3,4,5,6-} kla]perimidine (4d) (Table 5, entry 3). By a procedure identical with that described for the preparation of $\mathbf{4 b}, \mathbf{3 d}$ (30 $\mathrm{mg}, 0.068$ mmol) was converted into $4 \mathbf{d}(13 \mathrm{mg}, 53 \%)$ by the reaction with $\mathrm{Pd}(\mathrm{OAc})_{2}(1.5 \mathrm{mg}, 0.007 \mathrm{mmol}), \mathrm{PPh}_{3}(4.4 \mathrm{mg}, 0.017 \mathrm{mmol})$, and $\mathrm{K}_{3} \mathrm{PO}_{4}(28.7 \mathrm{mg}, 0.14 \mathrm{mmol})$ as red crystals: $\mathrm{mp} 283-$ $285{ }^{\circ} \mathrm{C} ;{ }^{1} \mathrm{H}$ NMR $\left(500 \mathrm{MHz}, \mathrm{CDCl}_{3}, 60{ }^{\circ} \mathrm{C}\right) \delta 6.60-6.62(\mathrm{~m}, 2 \mathrm{H}$, Ar), 6.87 (d, $J=8.0 \mathrm{~Hz}, 1 \mathrm{H}, \mathrm{Ar}), 7.06-7.16$ (m, 4H, Ar), 7.20 (dd, $J=8.3,8.3 \mathrm{~Hz}, 1 \mathrm{H}, \mathrm{Ar}), 7.32$ (dd, $J=7.7,7.7 \mathrm{~Hz}, 1 \mathrm{H}, \mathrm{Ar}), 7.52$ (d, $J=9.2 \mathrm{~Hz}, 1 \mathrm{H}, \mathrm{Ar}), 7.66(\mathrm{dd}, J=8.0,2.9 \mathrm{~Hz}, 1 \mathrm{H}, \mathrm{Ar}), 7.71(\mathrm{~d}$ $J=8.0 \mathrm{~Hz}, 1 \mathrm{H}$, Ar), $7.98(\mathrm{dd}, J=10.3,2.3 \mathrm{~Hz}, 1 \mathrm{H}, \mathrm{Ar}) ;{ }^{13} \mathrm{C}$ NMR assignment was difficult due to the poor solubility of $\mathbf{4 d}$ as well as C-F couplings; HRMS (FAB) calcd for $\mathrm{C}_{25} \mathrm{H}_{14} \mathrm{FN}_{2}$ $\left(\mathrm{MH}^{+}\right)$: 361.1141 ; found: 361.1140

4.4.5.

13-Methoxydibenzo[1,2:7,8]quinolizino[3,4,5,6-

kla]perimidine (4e) hydrochloride (Table 5, entry 4). Вy a procedure identical with that described for the preparation of $\mathbf{4 b}$, 3e $(30 \mathrm{mg}, 0.066 \mathrm{mmol})$ was converted into $4 \mathbf{e}(15 \mathrm{mg}, 61 \%)$ by the reaction with $\mathrm{Pd}(\mathrm{OAc})_{2}(1.5 \mathrm{mg}, 0.007 \mathrm{mmol}), \mathrm{PPh}_{3}(4.3 \mathrm{mg}$, $0.017 \mathrm{mmol})$, and $\mathrm{K}_{3} \mathrm{PO}_{4}(28.0 \mathrm{mg}, 0.13 \mathrm{mmol})$ as red crystals. The crystals were dissolved in $\mathrm{CHCl}_{3}$, and $4 \mathrm{~N}$ solution of $\mathrm{HCl}$ in dioxane was added to the mixture. Hexane was added to the mixture and the precipitates were collected by filtration to give 4e $\cdot \mathrm{HCl}$ as a brown solid: mp $140-141{ }^{\circ} \mathrm{C} ;{ }^{1} \mathrm{H}$ NMR $(500 \mathrm{MHz}$, $\left.\mathrm{CD}_{3} \mathrm{OD}\right) \delta 4.03\left(\mathrm{~s}, 3 \mathrm{H}, \mathrm{OCH}_{3}\right), 6.92(\mathrm{~d}, J=6.9 \mathrm{~Hz}, 1 \mathrm{H}, \mathrm{Ar}), 7.02$ (d, $J=8.0 \mathrm{~Hz}, 1 \mathrm{H}, \mathrm{Ar}), 7.09$ (dd, $J=7.4,7.4 \mathrm{~Hz}, 1 \mathrm{H}, \mathrm{Ar}), 7.34$ (m, 2H, Ar), 7.40 (dd, $J=7.4,7.4 \mathrm{~Hz}, 1 \mathrm{H}, \mathrm{Ar}), 7.47$ (d, $J=9.2$ $\mathrm{Hz}, 1 \mathrm{H}, \mathrm{Ar}), 7.67$ (d, $J=9.2 \mathrm{~Hz}, 1 \mathrm{H}, \mathrm{Ar}), 7.71$ (s, 1H, Ar), 7.79 (d, $J=9.2 \mathrm{~Hz}, 1 \mathrm{H}, \mathrm{Ar}), 7.85(\mathrm{~m}, 1 \mathrm{H}, \mathrm{Ar}), 7.91$ (s, 1H, Ar), 7.96 (d, $J=7.4 \mathrm{~Hz}, 1 \mathrm{H}, \mathrm{Ar}) ;{ }^{13} \mathrm{C} \mathrm{NMR}\left(125 \mathrm{MHz}, \mathrm{CD}_{3} \mathrm{OD}\right) \delta 57.3$, $104.5,110.5,110.9,117.9,118.1,120.8,123.1,123.6,123.9$, $125.7,126.1,127.7,127.8,128.1,129.1,131.0,131.3,132.7$, $132.8,132.9,134.1,134.4,135.7,148.7,162.6$; HRMS (FAB) calcd for $\mathrm{C}_{26} \mathrm{H}_{17} \mathrm{~N}_{2} \mathrm{O}\left(\mathrm{MH}^{+}\right)$: 373.1341 ; found: 373.1341 .

4.4.6. 14-Fluorodibenzo[1,2:7,8]quinolizino[3,4,5,6kla]perimidine (4f) hydrochloride (Table 5, entry 5). Вy a procedure identical with that described for the preparation of $\mathbf{4 b}$, 3f $(30 \mathrm{mg}, 0.068 \mathrm{mmol})$ was converted into $4 \mathrm{f}(13 \mathrm{mg}, 51 \%)$ by the reaction with $\mathrm{Pd}(\mathrm{OAc})_{2}(1.5 \mathrm{mg}, 0.007 \mathrm{mmol}), \mathrm{PPh}_{3}(4.4 \mathrm{mg}$, $0.017 \mathrm{mmol})$, and $\mathrm{K}_{3} \mathrm{PO}_{4}(28.7 \mathrm{mg}, 0.14 \mathrm{mmol})$ as red crystals. The crystals were dissolved in $\mathrm{CHCl}_{3}$, and $4 \mathrm{~N}$ solution of $\mathrm{HCl}$ in dioxane was added to the mixture. After hexane was added to the mixture, the precipitates were collected by filtration to give 4f $\cdot \mathrm{HCl}$ as a brown solid: mp $130-131{ }^{\circ} \mathrm{C} ;{ }^{1} \mathrm{H}$ NMR $(500 \mathrm{MHz}$, $\left.\mathrm{CD}_{3} \mathrm{OD}, 50{ }^{\circ} \mathrm{C}\right) \delta 6.75(\mathrm{~d}, J=7.4 \mathrm{~Hz}, 1 \mathrm{H}, \mathrm{Ar}), 7.07(\mathrm{~d}, J=8.0$ $\mathrm{Hz}, 1 \mathrm{H}, \mathrm{Ar}), 7.16$ (dd, $J=8.0,8.0 \mathrm{~Hz}, 1 \mathrm{H}, \mathrm{Ar}), 7.31-7.34(\mathrm{~m}, 2 \mathrm{H}$, Ar), 7.38 (dd, $J=7.4,7.4 \mathrm{~Hz}, 1 \mathrm{H}, \mathrm{Ar}), 7.44$ (dd, $J=14.0,7.7 \mathrm{~Hz}$, $1 \mathrm{H}, \mathrm{Ar}), 7.61$ (d, $J=8.0 \mathrm{~Hz}, 1 \mathrm{H}, \mathrm{Ar}), 7.81(\mathrm{~d}, J=9.2 \mathrm{~Hz}, 1 \mathrm{H}, \mathrm{Ar})$ 7.86-7.89 (m, 2H, Ar), 8.00 (s, $1 \mathrm{H}, A r), 8.07$ (d, $J=8.0 \mathrm{~Hz}, 1 \mathrm{H}$, Ar) ${ }^{13} \mathrm{C}$ NMR $\left(125 \mathrm{MHz}, \mathrm{CD}_{3} \mathrm{OD}, 50{ }^{\circ} \mathrm{C}\right) \delta 107.3(\mathrm{~d}, J=7.2 \mathrm{~Hz})$ $110.5,111.7,116.7(\mathrm{~d}, J=22.8 \mathrm{~Hz}), 117.6,120.6,123.2,123.9$, $124.4,125.2,125.7$ (d, $J=3.6 \mathrm{~Hz}), 126.4,128.1,128.3,129.5$, $131.2,131.5,133.1,133.6,135.5,136.7,138.6(\mathrm{~d}, J=10.8 \mathrm{~Hz})$, $139.7(\mathrm{~d}, J=8.4 \mathrm{~Hz}), 148.7,160.8(\mathrm{~d}, J=257.9 \mathrm{~Hz})$; HRMS (FAB) calcd for $\mathrm{C}_{25} \mathrm{H}_{14} \mathrm{FN}_{2}\left(\mathrm{MH}^{+}\right)$: 361.1141; found: 361.1143

\subsubsection{8-Fluorodibenzo[1,2:7,8]quinolizino[3,4,5,6-} kla]perimidine (4g) hydrochloride (Table 5, entry 6). By a procedure identical with that described for the preparation of $\mathbf{4 b}$,

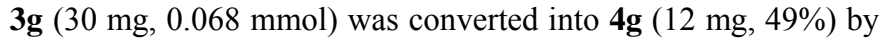
the reaction with $\mathrm{Pd}(\mathrm{OAc})_{2}(1.5 \mathrm{mg}, 0.007 \mathrm{mmol}), \mathrm{PPh}_{3}(4.4 \mathrm{mg}$, $0.017 \mathrm{mmol})$, and $\mathrm{K}_{3} \mathrm{PO}_{4}(28.7 \mathrm{mg}, 0.14 \mathrm{mmol})$ as red crystals. The crystals were dissolved in $\mathrm{CHCl}_{3}$, and $4 \mathrm{~N}$ solution of $\mathrm{HCl}$ in dioxane was added to the mixture. After hexane was added to the mixture, the precipitates were collected by filtration to give 4g. $\mathrm{HCl}$ as a brown solid: mp $280-281{ }^{\circ} \mathrm{C} ;{ }^{1} \mathrm{H}$ NMR $(500 \mathrm{MHz}$, $\left.\mathrm{CD}_{3} \mathrm{OD}, 60{ }^{\circ} \mathrm{C}\right) \delta 6.75(\mathrm{~d}, J=6.9 \mathrm{~Hz}, 1 \mathrm{H}, \mathrm{Ar}), 6.83(\mathrm{~d}, J=8.0$ $\mathrm{Hz}, 1 \mathrm{H}, \mathrm{Ar}$ ), 6.93-6.98 (m, 2H, Ar), 7.05 (d, $J=8.6 \mathrm{~Hz}, 1 \mathrm{H}, \mathrm{Ar})$, $7.46(\mathrm{~d}, J=9.2 \mathrm{~Hz}, 1 \mathrm{H}, \mathrm{Ar}), 7.55-7.58(\mathrm{~m}, 3 \mathrm{H}, \mathrm{Ar}), 7.64-7.67$ (m, $2 \mathrm{H}, \mathrm{Ar}), 7.75(\mathrm{dd}, J=7.4,7.4 \mathrm{~Hz}, 1 \mathrm{H}, \mathrm{Ar}), 8.25(\mathrm{~d}, J=8.0 \mathrm{~Hz}$, $1 \mathrm{H}, \mathrm{Ar}) ;{ }^{13} \mathrm{C} \mathrm{NMR}\left(125 \mathrm{MHz}, \mathrm{CD}_{3} \mathrm{OD}, 60{ }^{\circ} \mathrm{C}\right) \delta 110.9,111.6$, $111.9(\mathrm{~d}, J=25.2 \mathrm{~Hz}), 116.3,117.5,120.4,120.8(\mathrm{~d}, J=22.8$ $\mathrm{Hz}), 122.7,123.7,124.4,125.7,126.6(\mathrm{~d}, J=8.4 \mathrm{~Hz}), 127.6$ (d, $J$ $=8.4 \mathrm{~Hz}), 127.8,129.0,129.1,129.2,131.0,131.2,134.4,135.2$, $136.9,137.0,149.7,164.5(\mathrm{~d}, J=250.7 \mathrm{~Hz})$; HRMS (FAB) calcd for $\mathrm{C}_{25} \mathrm{H}_{14} \mathrm{FN}_{2}\left(\mathrm{MH}^{+}\right)$: 361.1141; found: 361.1141 .

4.4.8.

8-Methyldibenzo[1,2:7,8]quinolizino[3,4,5,6kla]perimidine (4h) hydrochloride (Table 5, entry 7). By a procedure identical with that described for the preparation of $\mathbf{4 b}$, 3h (30 mg, $0.068 \mathrm{mmol})$ was converted into $4 \mathbf{h}(15 \mathrm{mg}, 62 \%)$ by the reaction with $\mathrm{Pd}(\mathrm{OAc})_{2}(1.5 \mathrm{mg}, 0.007 \mathrm{mmol}), \mathrm{PPh}_{3}(4.5 \mathrm{mg}$, $0.017 \mathrm{mmol})$, and $\mathrm{K}_{3} \mathrm{PO}_{4}(29.0 \mathrm{mg}, 0.14 \mathrm{mmol})$ as red crystals. The crystals were dissolved in $\mathrm{CHCl}_{3}$, and $4 \mathrm{~N}$ solution of $\mathrm{HCl}$ in dioxane was added to the mixture. After hexane was added to the mixture, the precipitates were collected by filtration to give 4h $\cdot \mathrm{HCl}$ as a brown solid: $\mathrm{mp} 165-166{ }^{\circ} \mathrm{C} ;{ }^{1} \mathrm{H}$ NMR $(500 \mathrm{MHz}$, $\left.\mathrm{CD}_{3} \mathrm{OD}, 60^{\circ} \mathrm{C}\right) \delta 2.22(\mathrm{~s}, 3 \mathrm{H}, \mathrm{Ar}), 6.79(\mathrm{~d}, J=7.4 \mathrm{~Hz}, 1 \mathrm{H}, \mathrm{Ar})$, 6.89 (d, $J=8.0 \mathrm{~Hz}, 1 \mathrm{H}, \mathrm{Ar}), 7.00-7.04$ (m, 2H, Ar), 7.09 (d, $J=$ 8.6 Hz, 1H, Ar), 7.50-7.53 (m, 2H, Ar), 7.59-7.63 (m, 3H, Ar), 7.74-7.80 (m, 2H, Ar), 8.25 (d, $J=8.6 \mathrm{~Hz}, 1 \mathrm{H}, \mathrm{Ar}) ;{ }^{13} \mathrm{C}$ NMR $\left(125 \mathrm{MHz}, \mathrm{CD}_{3} \mathrm{OD}, 60{ }^{\circ} \mathrm{C}\right) \delta 21.6,110.4,110.9,116.7,117.3$, $120.5,122.7,123.6,123.8,125.2,125.5,125.7,127.9,128.9$, 129.1, 130.8, 131.0, 133.9 (2C), 134.3, 135.2, 135.5, 136.9, 137.5, 142.0, 149.7; HRMS (FAB) calcd for $\mathrm{C}_{26} \mathrm{H}_{17} \mathrm{~N}_{2}\left(\mathrm{MH}^{+}\right)$: 357.1392; found: 357.1390 .

\section{Acknowledgments}

This work was supported by a Grant-in-Aid for Scientific Research on Innovative Areas 'Integrated Organic Synthesis' (H.O.) and Targeted Proteins Research Program from the Ministry of Education, Culture, Sports, Science and Technology of Japan.

\section{References and notes}

1. Ahmed, E.; Earmme, T.; Ren, G.; Jenekhe, S. A. Chem. Mater. 2010, 22, 5786-5796.

2. Laali, K. K. Chem. Rev. 1996, 96, 1873-1906.

3. Scott, L. T.; Bronstein, H. E.; Preda, D. V.; Ansems, R. B. M.; Bratcher, M. S.; Hagen, S. Pure Appl. Chem. 1999, 71, 209-219.

4. Echavarren, A. M.; Gómez-Lor, B.; González, J. J.; de Frutos, Ó. Synlett 2003, 585-597.

5. Pascual, S.; de Mendoza P.; Echavarren, A. M. Org. Biomol. Chem. 2007, 5, 2727-2734.

6. For perimidine derivatives, see: Wu, Z.-A. G.; Haubrich, J. E.; Wang, X.; Liang, R.-C. Patent WO 2004010206. 
7. Herbert, J. M.; Woodgate, P. D.; Denny, W. A. J. Med. Chem. 1987, 30, 2081-2086.

8. Houlihan, W. J.; Cheon, S. H.; Parrino, V. A.; Handley, D. A.; Larson, D. A. J. Med. Chem. 1993, 36, 3098-3102.

9. Scholz, D.; Schmidt, H.; Prieschl, E. E.; Csonga, R.; Scheirer, W.; Weber, V.; Lembachner, A.; Seidl, G.; Werner, G.; Mayer, P.; Baumruker, T. J. Med. Chem. 1998, 41, 1050-1059.

10. Ohta, Y.; Kubota, Y.; Watabe, T.; Chiba, H.; Oishi, S.; Fujii, N.; Ohno, H. J. Org. Chem. 2009, 74, 6299-6302.

11. See also: Ohta, Y.; Oishi, S.; Fujii, N.; Ohno, H. Chem. Commun. 2008, 835-837.

12. Ames, D. E.; Bull, D. Tetrahedron 1982, 38, 383-387.

13. Ames, D. E.; Opalko, A. Tetrahedron 1984, 40, 1919-1925.

14. Zhang, M. Adv. Synth. Catal. 2009, 351, 2243-2270.

15. Thansandote, P.; Lautens, M. Chem. Eur. J. 2009, 15, 5874-5883.

16. For our recent work on C-H arylation, see: Ohno, H.; Miyamura, K.; Takeoka, Y.; Tanaka, T. Angew. Chem., Int. Ed. 2003, 42, 2647-2650.

17. Ohno, H.; Yamamoto, M.; Iuchi, M.; Tanaka, T. Angew. Chem., Int. Ed. 2005, 44, 5103-5106.

18. Ohno, H.; Miyamura, K.; Mizutani, T.; Kadoh, Y.; Takeoka, Y.; Hamaguchi, H.; Tanaka, T. Chem. Eur. J. 2005, 11, 3728-3741.

19. Watanabe, T.; Oishi, S.; Fujii, N.; Ohno, H. Org. Lett. 2007, 9, 4821-4824.

20. Ohno, H.; Iuchi, M.; Fujii, N.; Tanaka, T. Org. Lett. 2007, 9, 4813-4815.

21. Watanabe, T.; Oishi, S.; Fujii, N.; Ohno, H. Org. Lett. 2008, 10, 1759-1762.

22. Dyker, G.; Stirner, W.; Henkel, G. Eur. J. Org. Chem. 2000, 1433-1441.

23. Recently, a highly related copper-catalyzed halocyclization reaction was reported: Ouyang, H.-C.; Tang, R.-Y.; Zhong, P.; Zhang, X.-G.; Li, J.-H. J. Org. Chem. 2011, 76, 223-228.

24. Okamoto, N.; Sakurai, K.; Ishikura, M.; Takeda, K.; Yanada, R. Tetrahedron Lett. 2009, 50, 4167-4169.

25. Patil, N. T.; Mutyala, A. K.; Lakshmi, P. G. V. V.; Raju, P. V. K.; Sridhar, B. Eur. J. Org. Chem. 2010, 1999-2007.

26. For a related synthesis of fused perimidines by annulation of 1iminoisocoumarin derivatives with 1,8-diaminonaphthalene, see: Deady, L. W.; Rodemann, T. J. Heterocyclic Chem. 1998, 35, 1417-1419.

27. Bridges, A. J.; Lee, A.; Maduakor, E. C.; Schwartz, C. E. Tetrahedron Lett. 1992, 33, 7499-7502. 